\title{
Plasmodium falciparum Guanylyl Cyclase-Alpha and the Activity of Its Appended P4-ATPase Domain Are Essential for cGMP Synthesis and Blood-Stage Egress
}

\author{
Stephanie D. Nofal, ${ }^{\text {* }}$ Avnish Patel, ${ }^{a}$ Michael J. Blackman, ${ }^{\mathrm{a}, \mathrm{b}}$ Christian Flueck, ${ }^{\mathrm{a}}$ (D) David A. Baker ${ }^{\mathrm{a}}$ \\ aFaculty of Infectious and Tropical Diseases, London School of Hygiene and Tropical Medicine, London, United Kingdom \\ bMalaria Biochemistry Laboratory, The Francis Crick Institute, London, United Kingdom
}

ABSTRACT Guanylyl cyclases (GCs) synthesize cyclic GMP (cGMP) and, together with cyclic nucleotide phosphodiesterases, are responsible for regulating levels of this intracellular messenger which mediates myriad functions across eukaryotes. In malaria parasites (Plasmodium spp), as well as their apicomplexan and ciliate relatives, GCs are associated with a P4-ATPase-like domain in a unique bifunctional configuration. P4-ATPases generate membrane bilayer lipid asymmetry by translocating phospholipids from the outer to the inner leaflet. Here, we investigate the role of Plasmodium falciparum guanylyl cyclase alpha (GC $\alpha$ ) and its associated P4-ATPase module, showing that asexual blood-stage parasites lacking both the cyclase and P4-ATPase domains are unable to egress from host erythrocytes. GC $\alpha$-null parasites cannot synthesize cGMP or mobilize calcium, a cGMP-dependent protein kinase (PKG)-driven requirement for egress. Using chemical complementation with a cGMP analogue and point mutagenesis of a crucial conserved residue within the P4ATPase domain, we show that P4-ATPase activity is upstream of and linked to CGMP synthesis. Collectively, our results demonstrate that GC $\alpha$ is a critical regulator of PKG and that its associated P4-ATPase domain plays a primary role in generating cGMP for merozoite egress.

IMPORTANCE The clinical manifestations of malaria arise due to successive rounds of replication of Plasmodium parasites within red blood cells. Once mature, daughter merozoites are released from infected erythrocytes to invade new cells in a tightly regulated process termed egress. Previous studies have shown that the activation of cyclic GMP (cGMP) signaling is critical for initiating egress. Here, we demonstrate that $\mathrm{GC} \alpha$, a unique bifunctional enzyme, is the sole enzyme responsible for cGMP production during the asexual blood stages of Plasmodium falciparum and is required for the cellular events leading up to merozoite egress. We further demonstrate that in addition to the GC domain, the appended ATPase-like domain of GC $\alpha$ is also involved in CGMP production. Our results highlight the critical role of GC $\alpha$ in cGMP signaling required for orchestrating malaria parasite egress.

KEYWORDS ATPase, Plasmodium, cyclic GMP, malaria, signal transduction

ignaling through 3',5'-cyclic GMP (cyclic GMP [cGMP]) regulates innumerable celluIar processes across the animal kingdom, ranging from the control of cardiovascular function (reviewed in reference 1) and phototransduction (reviewed in reference 2) in mammals to differentiation and locomotion in several unicellular organisms (reviewed in reference 3). Two central players in the cGMP pathway are guanylyl cyclases (GCs), which synthesize cGMP from GTP, and phosphodiesterases (PDEs), which degrade cGMP. Cellular levels of cGMP are tightly regulated by the opposing action of these two enzyme classes. Upon reaching threshold levels, cGMP activates downstream effectors such as
Citation Nofal SD, Patel A, Blackman MJ, Flueck C, Baker DA. 2021. Plasmodium falciparum guanylyl cyclase-alpha and the activity of its appended P4-ATPase domain are essential for cGMP synthesis and blood-stage egress. mBio 12:e02694-20. https://doi.org/10.1128/mBio .02694-20.

Editor Louis H. Miller, NIAID/NIH

Copyright $\odot 2021$ Nofal et al. This is an openaccess article distributed under the terms of the Creative Commons Attribution 4.0 International license.

Address correspondence to David A. Baker, david.baker@lshtm.ac.uk.

*Present address: Stephanie D. Nofal, Signalling in Apicomplexan Parasites Laboratory, The Francis Crick Institute, London, United Kingdom.

Received 21 September 2020 Accepted 19 November 2020 Published 26 January 2021 
the cGMP-dependent protein kinase (PKG) or cGMP-gated ion channels. In the protozoan parasites responsible for malaria (Plasmodium spp.), PKG is thought to be the only direct effector of cGMP signaling since canonical cGMP-gated ion channels are absent from the genome (4). Malaria parasites have two GC paralogues which are structurally distinct from both types of mammalian GC (5-7). Each Plasmodium GC possesses a C-terminal domain with an overall topology similar to $G$ protein-dependent adenylyl cyclases, comprising two catalytic domains each preceded by a set of six transmembrane helices. These twin catalytic domains contain all of the conserved amino acid residues required for cGMP synthesis. However, while mammalian $G$ protein-dependent adenylyl cyclases are activated following binding of heterotrimeric $G$ proteins once a ligand binds to a $G$ protein-coupled receptor, $\mathrm{G}$ proteins are absent from the Plasmodium genome, suggesting an alternate mechanism of activation. In addition to the cyclase domain, Plasmodium GCs also possess an N-terminal domain that has high structural similarity to P4-ATPases or flippases $(5,8)$. In other organisms, flippases translocate phospholipids from the outer/luminal to the cytosolic leaflet of a membrane lipid bilayer, creating membrane asymmetry important for membrane remodeling, vesicular transport and signaling (reviewed in references 9-11). Most phospholipid flippases function in partnership with an integral membrane Cell Division Control protein 50 (CDC50), which acts as a chaperone for the enzyme and is required for flippase activity $(12,13)$. The physical linkage of a guanylyl cyclase with a P4-ATPase domain is unique to Plasmodium and its apicomplexan and ciliate relatives $(5,8,14-17)$. Recent work in Toxoplasma gondii has investigated the role of the single GC isoform in parasite development. The Toxoplasma GC (TgGC) localizes to the plasma membrane at the basal and apical ends of invasive tachyzoites (15-18). Conditional knockdown of TgGC revealed that it is responsible for cGMP production, which is required for parasite egress, calcium release, microneme exocytosis, motility, host cell attachment, and invasion (15-18). TgGC requires interaction with a CDC50 protein and a unique GC organizer, which act as chaperones for the ATPase and GC domains, respectively (14). The ATPase domain is required for targeting TgGC to the apical end of the parasite (15) and mutagenesis of key residues required for ATPase activity inhibit microneme release and lytic growth $(14,15)$. GC-mediated phospholipid flipping activity could not be detected (14); however, there is evidence that $\mathrm{TgGC}$ is involved in sensing extracellular $\mathrm{pH}$ and $\mathrm{K}^{+}$levels (17), as well as phosphatidic acid (PA) (14).

The complex life cycle of the malaria parasite is divided between a vertebrate host and a mosquito vector, and there is abundant evidence that cGMP signaling is crucial for parasite survival across multiple developmental stages. While the asexual blood stages are solely responsible for clinical disease in the vertebrate host, a crucial step for transmission to the vector is the generation of sexual gametocyte forms. Upon uptake in a blood meal, the gametocytes are activated by a combination of reduced temperature with either an increase in $\mathrm{pH}$ or the presence of the mosquito factor xanthurenic acid (XA) (19), triggering the emergence of male and female gametes (gametogenesis). An early study using pharmacological agents suggested a role for CGMP signaling in male gamete activation in the mosquito (61), and the action of XA was subsequently linked to elevated cGMP levels in vitro (20). Using chemical genetic approaches, we showed that CGMP signaling through PKG is essential for gametogenesis (21) and also for gliding motility of ookinetes (22), motile forms that develop from the zygote that results from gamete fertilization. Of the two $P$. falciparum GC isoforms, GC $\beta$ is dispensable for blood-stage development and is expressed exclusively in the mosquito stages (23), where it is required for CGMP synthesis to drive and maintain $P$. berghei ookinete gliding motility $(22,24,25)$. GC $\beta$ becomes polarized during ookinete formation, is stabilized by a CDC50 protein, and is thought to elevate local levels of cGMP to facilitate gliding motility (8). The ookinetes penetrate the mosquito midgut epithelium and form oocysts in which thousands of sporozoites form; these then migrate to and invade the mosquito salivary glands. Dysregulation of cGMP levels by deletion of phosphodiesterase-gamma (PDE $\gamma$ ) in the rodent malaria species Plasmodium yoelii blocks salivary gland 
invasion and subsequent parasite development (26). In another rodent malaria parasite, $P$. berghei, PKG is required for sporozoite motility and invasion of hepatocytes following injection of sporozoites into a host by the mosquito (27). Furthermore, conditional disruption of PKG reduces the release of merosomes containing merozoites into the bloodstream prior to erythrocyte invasion (28).

In contrast to $\mathrm{GC} \beta$, transcriptomic data indicate that $P$. falciparum $\mathrm{GC} \alpha$ is expressed in both gametocytes and the asexual blood stages of the life cycle (https://plasmodb .org/plasmo/). The GC $\alpha$ gene has so far proved refractory to disruption in asexual blood stages $(23,25,29)$, consistent with an important role during this clinically relevant stage. Confirmation of a key role for CGMP signaling in blood stages was obtained using a chemical genetic approach which demonstrated that PKG is essential for schizont rupture (30). This observation was subsequently extended to show that just prior to merozoite egress, PKG regulates the discharge of the subtilisin-like protease SUB1 from organelles termed exonemes into the parasitophorous vacuole (PV) of mature $P$. falciparum schizonts, where it proteolytically processes a number of proteins required for merozoite egress and invasion $(31,32)$. PKG activity is also required for the release of calcium ions $\left(\mathrm{Ca}^{2+}\right)$ from internal stores that is a prerequisite for egress (22). In efforts to dissect the mechanistic basis of this PKG-dependent egress pathway, comparative phosphoproteomic analysis identified 69 proteins that are phosphorylated in a cGMP-dependent manner (33). Very recently, the essential role for PKG in egress was confirmed through conditional genetic approaches (34). Despite this clear evidence for a crucial role for PKG in the asexual blood-stage life cycle, the role of GC $\alpha$ and its appended P4-ATPase domain remains unexplored.

Here, we show that GC $\alpha$ is essential for P. falciparum blood-stage egress and that GC $\alpha$-null parasites cannot synthesize cGMP or mobilize $\mathrm{Ca}^{2+}$. Crucially, we show that activity of the P4-ATPase domain of GC $\alpha$ is essential and that it functions upstream of cGMP synthesis.

\section{RESULTS}

$P$. falciparum $\mathrm{GC} \alpha$ is expressed during late asexual blood-stage development and localizes to cytoplasmic vesicular structures in newly formed merozoites. Upon invasion of a red blood cell by a malaria merozoite, the parasite transforms through ring and trophozoite stages and then undergoes DNA replication to form a multinucleated schizont that eventually segments to form a new generation of daughter merozoites. These are then released upon egress to invade fresh red cells and repeat the cycle. Invasion is driven by an actinomyosin-based contractile complex often referred to as the glideosome, which lies beneath the pellicular membrane of the merozoite. Data from several previous transcriptome studies show that GC $\alpha$ mRNA expression peaks at the schizont stage, while proteomic analysis indicates presence of the protein in schizonts and merozoites (http://plasmodb.org/). To define the timing of expression of GC $\alpha$ at the protein level and to determine its subcellular localization, we generated a $P$. falciparum line expressing GC $\alpha$ fused to a C-terminal triple hemagglutinin $(3 \times \mathrm{HA})$ epitope tag. For this, we used the $P$. falciparum $1 \mathrm{G} 5$ clone, which constitutively expresses a dimerizable Cre recombinase (DiCre) that can be activated by treatment with rapamycin (RAP) (35). The tagging strategy used single crossover homologous recombination at the $3^{\prime}$ end of the endogenous GC $\alpha$ gene, along with introduction of a human dihydrofolate reductase (hDHFR) selection cassette flanked by two loxP sites (Fig. 1A). Initial selection for transformants with the antifolate WR99210, followed by several rounds of drug cycling, enriched for parasites in which integration had occurred. The hDHFR selectable marker was then recycled by RAP-induced Cre recombinase-mediated excision of the floxed sequence, leaving behind a single lox $P$ site immediately downstream of the $3 \times \mathrm{HA}$ epitope tag and translational stop codon (Fig. 1A). Two parasite clones were obtained that showed sensitivity to WR99210, suggesting the expected genomic rearrangement with loss of the hDHFR selection marker (Fig. 1B). The absence of the hDHFR cassette was confirmed in GC $\alpha$ :HA clone 1 by PCR 
A

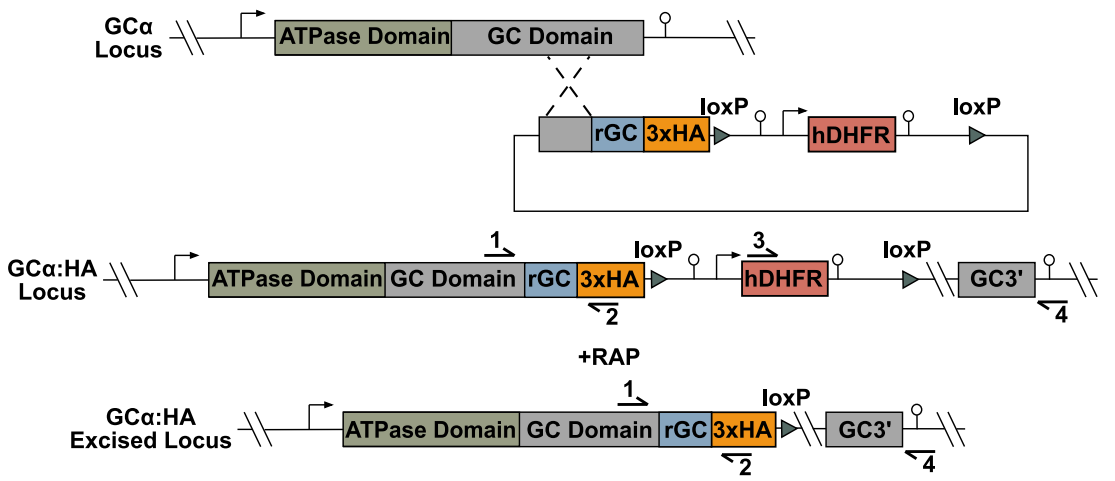

B

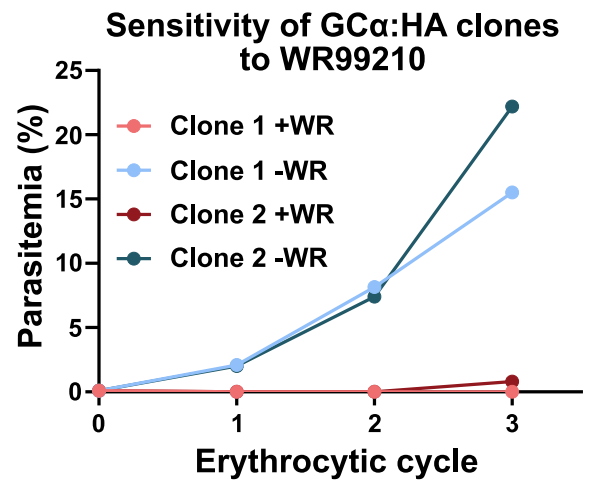

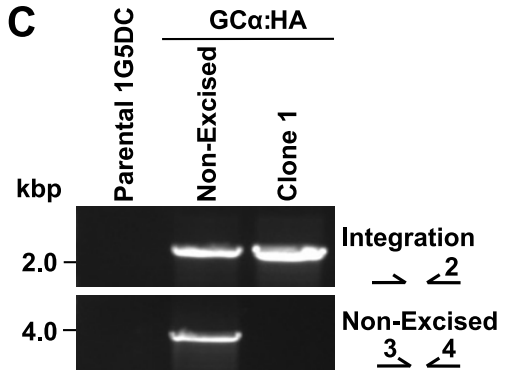

E

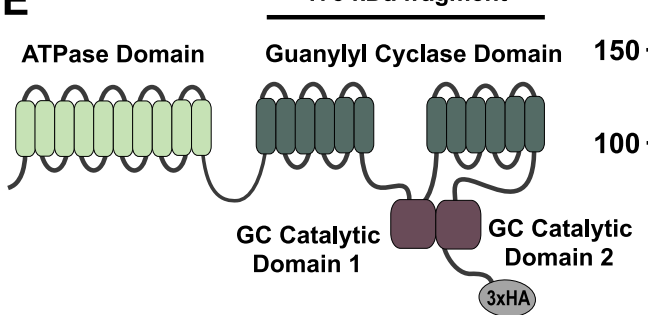

D
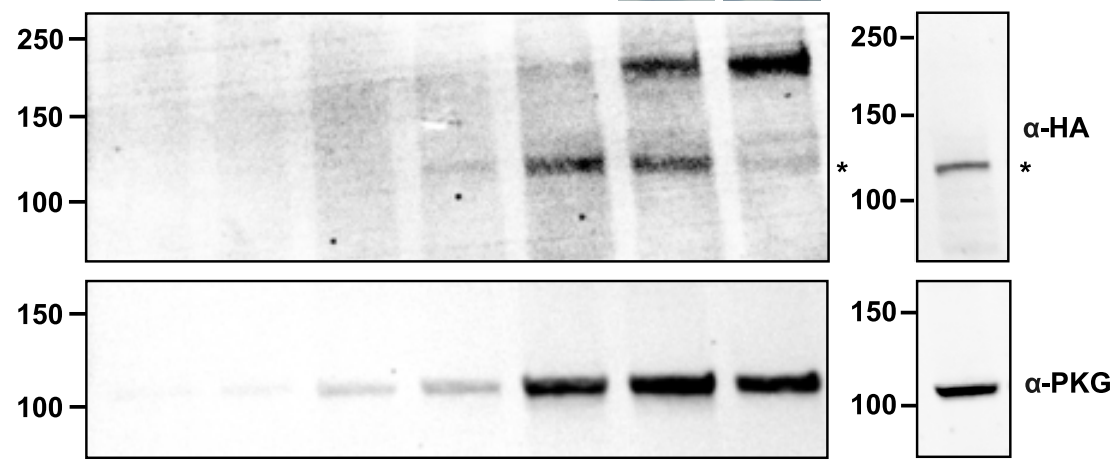

$\mathbf{F}$

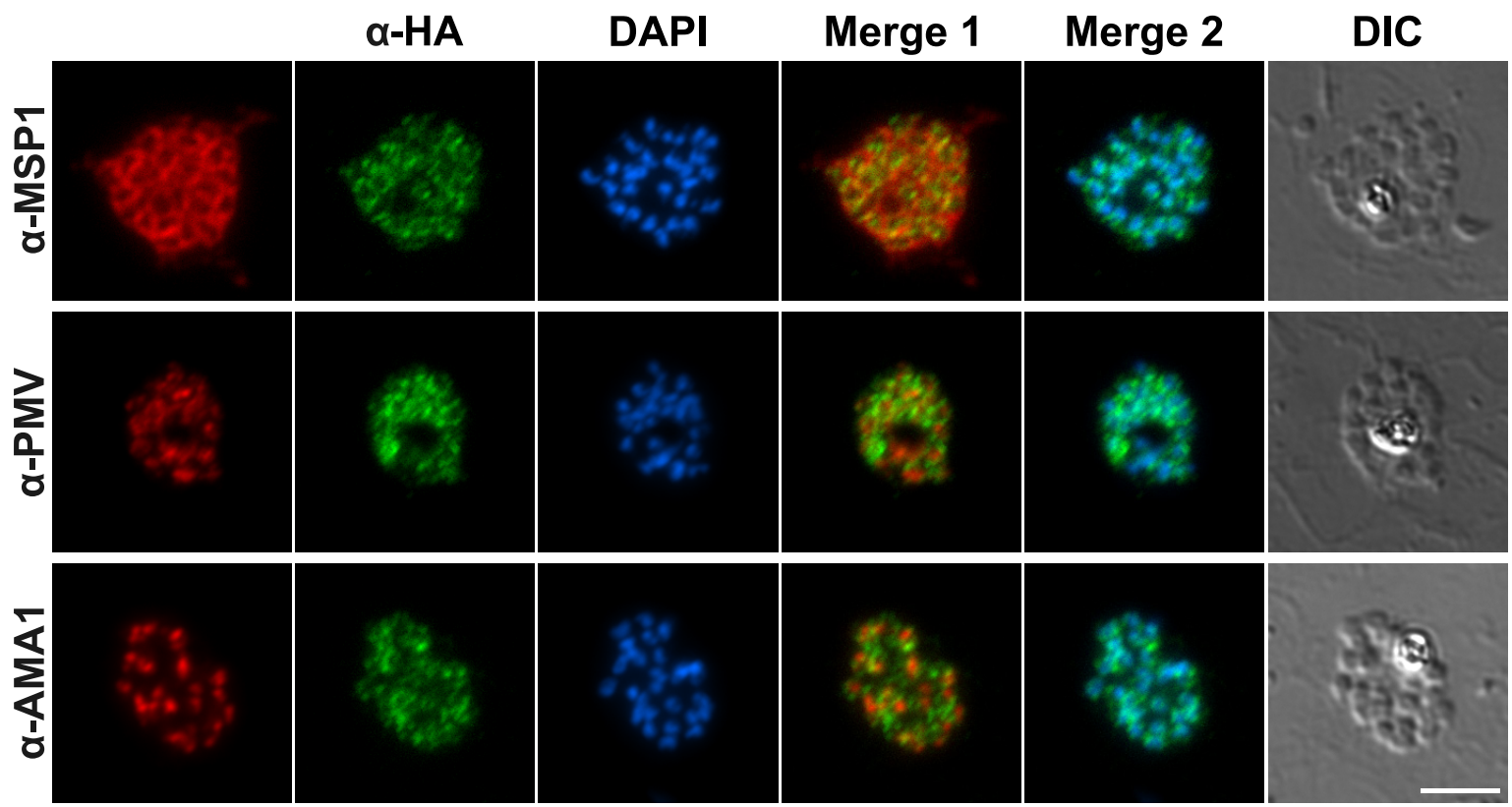

FIG 1 Generation of a GC $\alpha$ :HA-tagged line and spatiotemporal expression of GC $\alpha$ in $P$. falciparum blood stages. (A) Schematic representation of the single homology crossover approach used to fuse the $3^{\prime}$ end of the endogenous GC $\alpha$ gene to a $3 \times H A$ tag, and subsequent RAP-mediated excision of (Continued on next page) 
analysis (Fig. 1C), and this transgenic parasite clone (referred to as GC $\alpha$ :HA) was subsequently used in all further experiments.

Tightly synchronized cultures of the GC $\alpha$ :HA line were sampled at 4-h intervals from the early trophozoite stage ( $24 \mathrm{~h}$ postinvasion) to the mature schizont stage ( $48 \mathrm{~h}$ postinvasion) and analyzed by Western blotting using $\alpha$-HA antibodies. This revealed a single specific signal migrating at $\sim 175 \mathrm{kDa}$ that was most intense in mature schizonts (Fig. 1D). This molecular mass is consistent with a C-terminal GC $\alpha-3 \times \mathrm{HA}$ fragment predicted to comprise both the guanylyl cyclase catalytic domains ( $\mathrm{C} 1$ and $\mathrm{C} 2$ ) plus the 12 associated transmembrane helices (Fig. 1E). No signal was detectable at the $\sim 500-\mathrm{kDa}$ mass expected for the full-length protein.

To confirm the timing of GC $\alpha$ expression at the single cell level and to reveal its subcellular localization, we performed dual staining immunofluorescence microscopy (IFA) with anti-HA in combination with known markers for different subcellular compartments. In mature GC $\alpha$ :HA schizonts GC $\alpha$ localized to intracellular foci, but not the plasma membrane as established by costaining with a merozoite surface protein 1 (MSP1) antibody (Fig. 1F, top panel). To further characterize the nature of the intracellular compartment occupied by $\mathrm{GC} \alpha$, we costained with antibodies that react with apical membrane antigen 1 (AMA1), a micronemal marker, or plasmepsin $\mathrm{V}$, an endoplasmic reticulum-resident protein. The $\alpha$-HA staining showed no significant overlap with either of these markers, nor with a nuclear stain (Fig. 1F, middle and bottom panels). We conclude that GC $\alpha$ localizes to non-apical, cytoplasmic vesicular structures and is maximally expressed in mature schizonts.

GC $\alpha$ is essential for asexual blood-stage growth and merozoite egress. To investigate the function and essentiality of $\mathrm{GC} \alpha$, we used the DiCre recombinase system (35) to inducibly disrupt the GC $\alpha$ gene. For this, we created a GC $\alpha$ conditional knockout (cKO) line by introducing a second loxP site into the GC $\alpha$ locus of the GC $\alpha$ :HA line. This additional loxP site was incorporated within an artificial SERA2 intron (loxPint) (36) inserted into the ATPase domain of GC $\alpha$ using marker-free CRISPR/Cas9-mediated gene editing (see Fig. S1A in the supplemental material). Integration of the loxPint into the $\mathrm{GC} \alpha$ coding region was confirmed by PCR in the uncloned parasite population (see Fig. S1B) and, following limiting-dilution cloning, two clones were obtained with the desired modification (see Fig. S1C). One of these clones, called GC $\alpha$ :HA:cKO, was used in all further experiments.

Activation of Cre recombinase in the GC $\alpha$ :HA:cKO line by the addition of RAP was expected to excise DNA sequences encoding part of the ATPase domain and the entire cyclase domain of GC $\alpha$, abrogating both enzymatic activities (Fig. 2A). To examine the efficiency of excision, synchronous cultures of young ring-stage parasites were treated with either $50 \mathrm{nM}$ RAP or the equivalent concentration of vehicle only (dimethyl sulfoxide [DMSO]) for $2 \mathrm{~h}$ and allowed to mature to the schizont stage. PCR analysis showed highly efficient RAP-induced excision (Fig. 2B), while Western blot analysis showed

FIG 1 Legend (Continued)

the hDHFR cassette. Promoters/5' untranslated regions (UTRs) are indicated by arrows, and 3' UTRs/terminators are indicated by lollipops. Triangles represent loxP sites; arrows with numbers represent the relative position of oligonucleotide primers used for diagnostic PCR. rGC $\alpha$ refers to recodonized $G C \alpha$ gene sequences. (B) Growth curve for two cloned $G C \alpha$ :HA lines sensitive to treatment with $2.5 \mathrm{nM}$ WR99210, consistent with successful excision of the hDHFR cassette. The data presented are from counting parasites on Giemsa-stained blood smears. At least 100 parasites were counted per condition. Clone 1 was used for all further experiments. (C) Diagnostic PCR analysis confirming successful integration of the $3 \times \mathrm{HA}$ tag and efficient excision of the floxed hDHFR cassette in GC $\alpha: H A$ clone 1. Lane 1, parental 1G5DC; lane 2, GC $\alpha: H A$ nonexcised; lane 3, GC $\alpha$ :HA clone 1 (excised). (D) Western blot showing a time course of $\mathrm{GC} \alpha$ :HA expression in P. falciparum blood stages. Parasites were harvested from cultures synchronized to a 2-h invasion window at the times indicated, with representative microscopy images shown above each sample (hpi, hours postinvasion). Scale bar, $5 \mu \mathrm{m}$. Blots were probed with a monoclonal $\alpha$-HA antibody to visualize the GC $\alpha$ :HA fusion protein and an anti-PKG antibody as a staging control. GC $\alpha$ :HA migrated as an $\sim 175-\mathrm{kDa}$ fragment, while full-length protein (predicted $\sim 499 \mathrm{kDa}$ ) could not be detected. Note that the additional band at $\sim 125 \mathrm{kDa}\left({ }^{*}\right)$ arises from a cross-reactivity of the $\alpha$-HA antibody with an unrelated parasite protein, since it is also detected in extracts from the unmodified 1G5 parental $P$. falciparum line (right panel). (E) Schematic representation of the domain architecture of GC $\alpha$, showing the N-terminal ATPase domain and the C-terminal guanylyl cyclase domain. The horizontal line with corresponding molecular mass shows the features likely contained in the C-terminal GC $\alpha$-HA fragment detected by Western blotting. (F) Dual staining IFA analysis of mature GC $\alpha$ :HA schizonts. Formaldehyde-fixed thin films were stained with $\alpha$-HA (green) and costained with antibodies to markers for known subcellular compartments (red): MSP1 (parasite plasma membrane, top panel), AMA1 (micronemes, middle panel), and plasmepsin V (endoplasmic reticulum, bottom panel). Scale bar $5 \mu \mathrm{m}$. For additional images, see Fig. S2A in the supplemental material. 

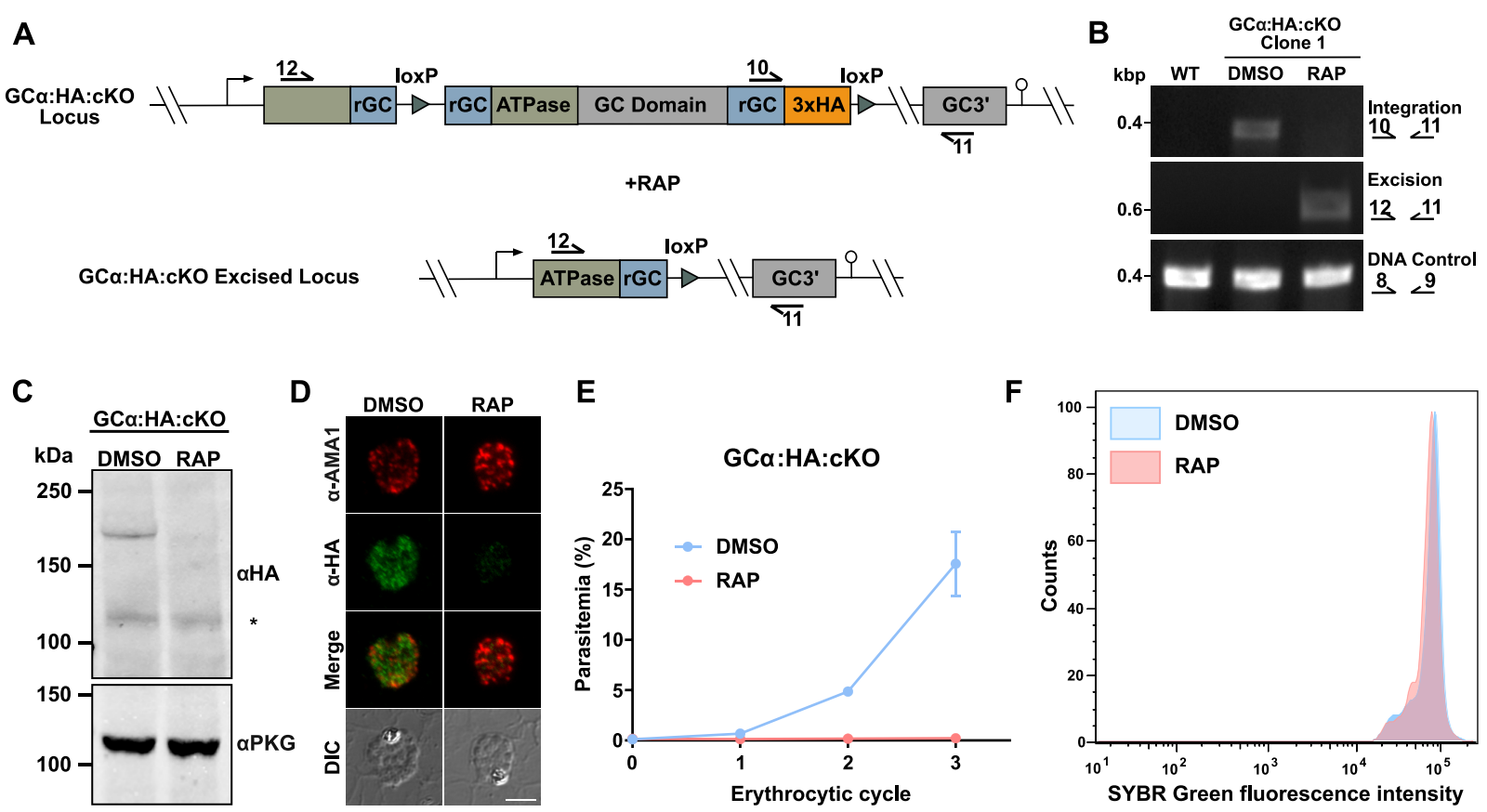

G

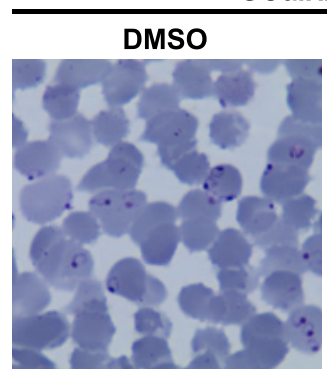

GCa:HA:cKO

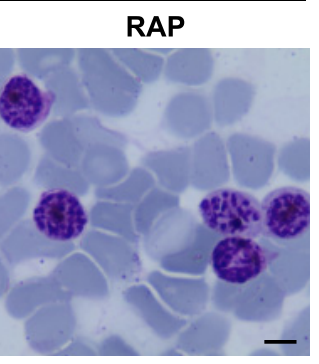

H

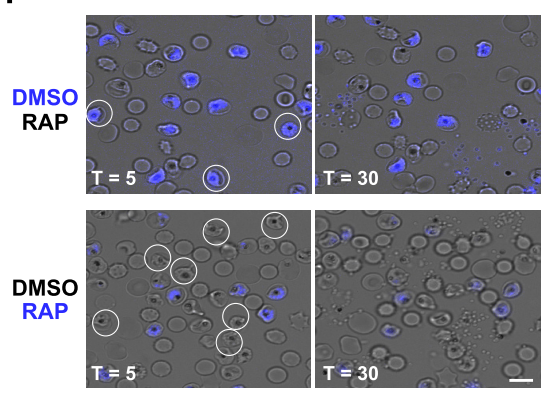

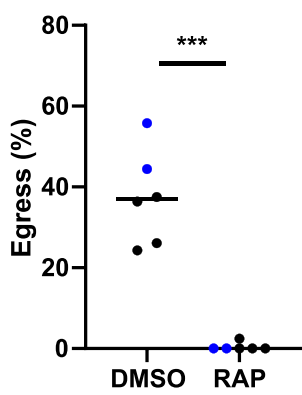

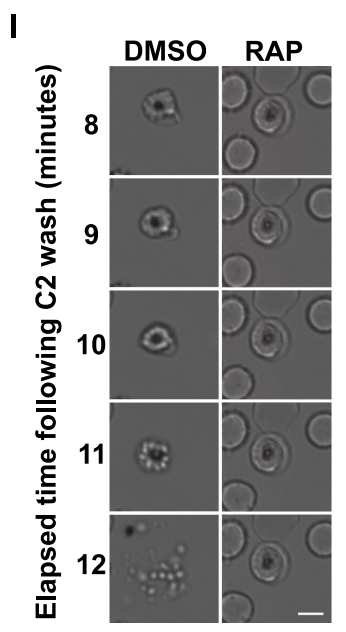

J
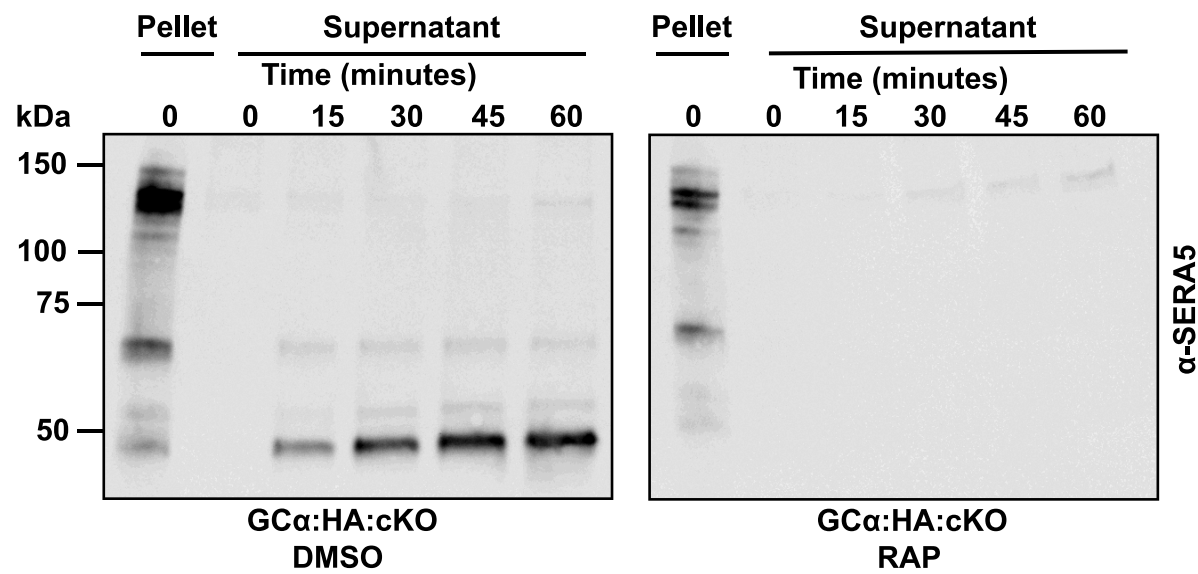

FIG 2 Efficient conditional disruption of GC $\alpha$ results in a complete egress block. (A) Schematic representation of RAP-induced excision of a portion of the ATPase domain and the entire GC domain of GC $\alpha$ in GC $\alpha$ :HA:cKO. Arrows represent the relative position of oligonucleotide primers used for diagnostic PCR screens. (B) Diagnostic PCR analysis of DMSO- and RAP-treated GC $\alpha$ :HA:cKO parasites showing efficient excision of the GC $\alpha$ gene following RAP treatment. A DNA control PCR was included, which amplified a small segment at an independent locus (Pf3D7_1342600) to confirm the quality of the DNA used. The specific PCR primers used are indicated on the right, and their binding sites are shown in panel A. (C) Western blot analysis of DMSO- and RAP-treated GC $\alpha$ :HA:cKO schizont lysates probed with an $\alpha$-HA antibody, showing loss of the $\sim 175-\mathrm{kDa}$ band in the RAP-treated sample, while the nonspecific $\sim 125-\mathrm{kDa}\left({ }^{*}\right)$ band can still be observed in both

(Continued on next page) 
complete loss of the $175-\mathrm{kDa}$ GC $\alpha-3 \times \mathrm{HA}$ signal in the RAP-treated GC $\alpha$ :HA:cKO schizonts, confirming efficient gene disruption (Fig. 2C). In addition, immunofluorescence microscopy showed loss of GC $\alpha-3 \times \mathrm{HA}$ at the single cell level (Fig. 2D; see also Fig. S2B and $\mathrm{C}$ ).

To assess the impact of GC $\alpha$ disruption on parasite viability and growth, we used flow cytometry to assess the replication of DMSO-treated (control) and RAP-treated GC $\alpha$ :HA:cKO parasites over three erythrocytic growth cycles. This revealed a complete growth arrest resulting from disruption of the GC $\alpha$ locus (Fig. 2E). Examination of Giemsa-stained GC $\alpha$ :HA:cKO parasites in the cycle of gene excision (cycle 0 ) revealed that RAP-treated cultures developed normally and were able to form mature segmented schizonts with a DNA content indistinguishable from control schizonts (Fig. 2F). However, upon further incubation we observed an accumulation of schizonts and a complete absence of newly formed ring-stage parasites, indicating that GC $\alpha$ is required for egress (Fig. 2G). To further characterize and quantify the egress defect in the GC $\alpha$-null parasites, highly synchronized mature cycle 0 schizonts from DMSO- and RAP-treated cultures were monitored by live time-lapse microscopy. This confirmed that $\mathrm{GC} \alpha$-null parasites were unable to egress (Fig. $2 \mathrm{H}$ ). Close examination of individual frames of the time-lapse video series revealed no signs of swelling or PVM rupture in GC $\alpha$-null schizonts (Fig. 2l). Consistent with the above results, the abundant PV-resident protein SERA5, which is released during egress, was not detectable in culture supernatant samples from RAP-treated cultures (Fig. 2J). Collectively, these results establish that GC $\alpha$ plays an essential role in the asexual blood-stage life cycle and is required for merozoite egress.

GC $\alpha$ is responsible for CGMP production and essential for calcium release in asexual blood-stage schizonts. The phenotypic similarity between the egress block observed in GC $\alpha$-null schizonts and that produced by the PKG inhibitor compound 2 (Fig. 2 l and Fig. 3A) is consistent with GC $\alpha$-null schizonts failing to activate PKG due to a lack of cGMP synthesis. To evaluate the impact of GC $\alpha$ disruption on cyclic nucleotide production, we compared intracellular cGMP concentrations in mature cycle 0 GC $\alpha$ :HA: cKO schizonts from DMSO- or RAP-treated cultures. For these experiments, in order to prevent egress of the DMSO-treated (control) schizonts, both sets of parasites under comparison were maintained in the presence of compound 2. This analysis showed that ablation of GC $\alpha$ led to a $94.5 \%$ reduction in cGMP levels compared to control parasites (Fig. 3B). Treatment with the PDE inhibitor zaprinast, which blocks CGMP hydroly-

FIG 2 Legend (Continued)

samples. The blots were also probed with an $\alpha$-PKG antibody to serve as a loading control. (D) Parallel IFA analysis of mature schizonts from DMSO- and RAP-treated GC $\alpha$ :HA:cKO cultures shows loss of GC $\alpha$-3xHA protein at the single cell level. Formaldehyde-fixed thin films were probed with $\alpha$-HA (green) and $\alpha$-AMA1 (red) antibodies. Scale bar, $5 \mu \mathrm{m}$. This analysis also confirms the specificity of the signal obtained with anti-HA. Additional images are shown in Fig. S2B and C. (E) Growth curves showing parasitemia of GC $\alpha$ :HA:cKO cultures treated with either DMSO or RAP, measured over three replication cycles by flow cytometry-based counting of SYBR green-positive cells. Data points plotted are means from two repeat experiments, each performed in triplicate. Error bars indicate the standard deviations. Note that from $\sim 16$ days after RAP treatment parasites emerged, but these were evidently not GC $\alpha$-null since PCR showed that the GC $\alpha$ locus was intact (see Fig. S3A and B). (F) Comparison of the DNA content in wild-type and GC $\alpha$-null schizonts. Schizonts obtained from synchronous DMSOand RAP-treated GC $\alpha$ :HA:cKO cultures were arrested using compound 2 to prevent egress and fixed at $\sim 46 \mathrm{~h}$ postinvasion, and parasite DNA was stained with SYBR green. SYBR green fluorescence intensity was measured by FACS counting from technical triplicates. (G) Representative microscopy images of Giemsa-stained parasites from DMSO- and RAP-treated GC $\alpha$ :HA:cKO cultures at $\sim 50 \mathrm{~h}$ postinvasion, showing the accumulation of schizonts in the RAP-treated culture while new ring-stage parasites formed in the DMSO control culture. Scale bar, $5 \mu \mathrm{m}$. (H) Combined DIC and fluorescence images from time-lapse video microscopy of DMSO- and RAP-treated GC $\alpha$ :HA:cKO schizonts taken at $5 \mathrm{~min}(T=5)$ and $30 \mathrm{~min}(T=30)$ after release from a compound 2 block applied to transiently prevent and subsequently synchronize egress. In each experiment, one subset of parasites was pretreated with Hoechst to stain the nuclei so that DMSO- and RAPtreated parasites could be viewed simultaneously in the same imaging chamber. In the top panel, DMSO-treated parasites are Hoechststained, while in the bottom panel, RAP-treated parasites are Hoechst-stained. Schizonts visible in the first frame that rupture over the course of the video are circled in white. Scale bar, $10 \mu \mathrm{m}$. The graph on the right shows a quantification of the percentage of DMSO- and RAPtreated schizonts that egressed in each 30-min video. Data were collected from six videos, with Hoechst-treated samples depicted in blue. Statistical significance was measured by unpaired $t$ test, where "***" signifies $P<0.0001$. (I) Time series of individual stills from GC $\alpha$ :HA:cKO time-lapse video microscopy in panel G. Images following representative schizonts for each condition (DMSO and RAP) from 8 to 12 min after compound 2 washout are shown. Note that the PVM around the RAP-treated schizont remains intact throughout the time series, whereas the DMSO control has completed egress by $12 \mathrm{~min}$. Scale bar, $5 \mu \mathrm{m}$. (J) Western blot analysis monitoring the release of SERA5 $(50 \mathrm{kDa})$ into the culture supernatant of DMSO- and RAP-treated GC $\alpha: \mathrm{HA}: \mathrm{cKO}$ schizonts, as a measurement of egress over time. Sampling times are indicated in minutes. Pellet samples were included as loading controls for full-length SERA5 (120 kDa). 
A

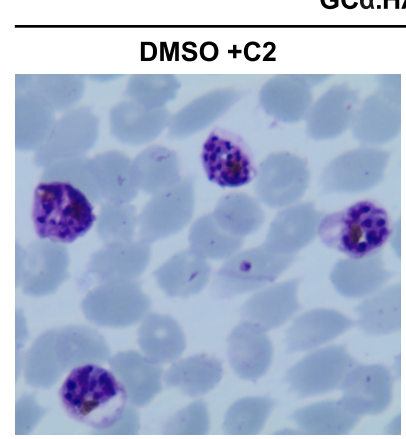

GCa:HA:cKO

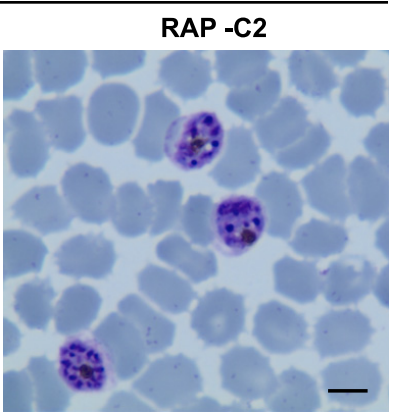

B

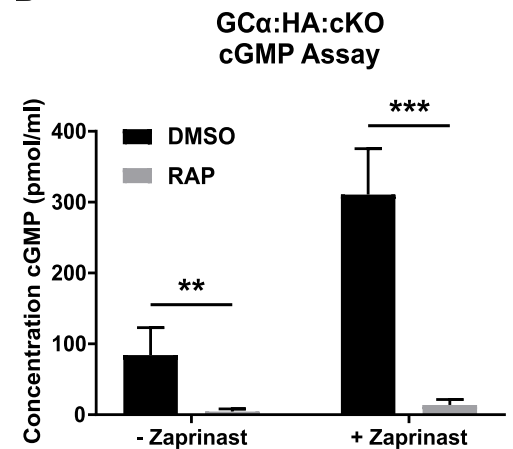

C

GCa:HA:cKO

cAMP Assay

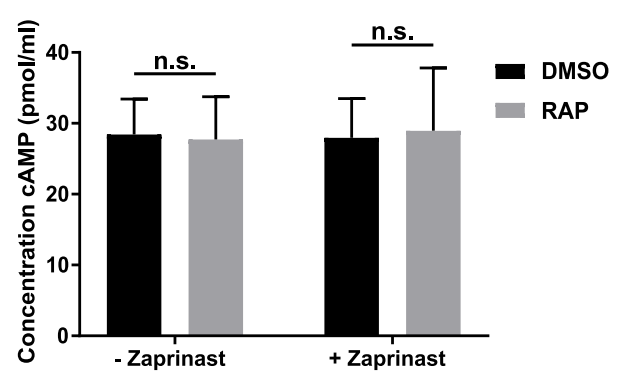

D

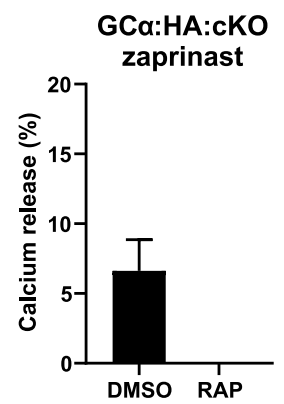

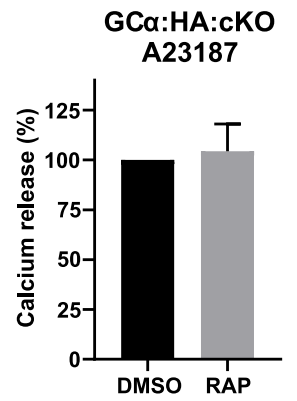

FIG 3 GC $\alpha$ disruption leads to a complete loss of cGMP production and a block in PKG-mediated calcium release. (A) Representative images of Giemsa-stained schizonts from DMSO-treated GC $\alpha$ :HA:cKO parasites in the presence of $1.5 \mu \mathrm{M}$ compound 2 and RAP-treated GC $\alpha$ :HA:cKO parasites at $50 \mathrm{~h}$ postinvasion. PKG inhibition leads to a similar phenotype to that observed in GC $\alpha$-null parasites. Scale bar, $5 \mu \mathrm{m}$. (B) Comparison of intracellular cGMP levels in wildtype and GC $\alpha$-null schizonts. Schizonts from DMSO- and RAP-treated GC $\alpha$ :HA:cKO cultures were matured in the presence of $1.5 \mu \mathrm{M}$ compound 2 to prevent egress. Segmented schizonts were either lysed directly (- zaprinast) or pretreated with $75 \mu \mathrm{M}$ zaprinast for 3 min prior to cell lysis (+ zaprinast). All samples were lysed in $0.1 \mathrm{M} \mathrm{HCl}$ to inactivate all PDEs, and extracts were analyzed by using a commercial ELISA-based CGMP detection assay. The data presented are means from four independent experiments, each assayed in duplicate. Error bars indicate the standard deviations. Statistical significance was measured by unpaired $t$ test $\left({ }^{* *}, P<0.01 ;{ }^{* * *}, P<0.001\right)$. (C) The samples described in panel B were assayed in parallel for CAMP with an ELISA-based CAMP kit from the same manufacturer. The data presented are means from four independent experiments, each performed in duplicate. Error bars indicate the standard deviations. Statistical significance was measured by unpaired $t$ test (n.s., not significant). (D) Determination of PKG-mediated calcium release from internal stores in wild-type and GC $\alpha$ KO schizonts. Fluo-4-loaded mature DMSO- and RAP-treated GC $\alpha$ :HA:cKO schizonts were treated with $75 \mu \mathrm{M}$ zaprinast (left panel) or $20 \mu \mathrm{M}$ ionophore A23187 (right panel), and the mobilization of $\mathrm{Ca}^{2+}$ was measured by fluorimetry. Zaprinast signals (left plot) were normalized to their respective ionophore control. lonophore signals were normalized to the DMSO-treated sample (right plot). Data plotted are mean values from two independent experiments performed in triplicate. Error bars indicate the standard deviations.

sis in P. falciparum schizonts (31) and thus artificially elevates CGMP levels, resulted in a significant increase in CGMP levels in the DMSO- but not the RAP-treated samples, confirming that $\mathrm{GC} \alpha$-null schizonts are unable to synthesize cGMP (Fig. 3B). Importantly, we detected no significant differences in cAMP levels between the control and GC $\alpha$ null parasites, nor did cAMP levels increase upon zaprinast treatment (Fig. 3C), consistent with our previous findings (31).

Next, we investigated whether $\mathrm{Ca}^{2+}$ release from internal stores into the cytosol, a PKG-dependent process that is essential for merozoite egress (22), was impaired in the GC $\alpha$ knockout parasites. Treatment with the PDE inhibitor zaprinast specifically induces PKG-mediated calcium release (22). We measured zaprinast-induced $\mathrm{Ca}^{2+}$ release in DMSO- and RAP-treated GC $\alpha$ :HA:cKO parasites by loading cells with the fluorescent calcium indicator Fluo-4 AM. As shown in Fig. 3D, while zaprinast treatment stimulated elevated cytosolic $\mathrm{Ca}^{2+}$ levels in control schizonts no such elevation of $\mathrm{Ca}^{2+}$ levels was detected in the GC $\alpha$-null schizonts (Fig. 3D). Importantly, both control and GC $\alpha$-null schizonts showed similar response levels to the calcium ionophore A23187, which 
allows $\mathrm{Ca}^{2+}$ ions to cross cell membranes, indicating that internal calcium stores were not affected in the absence of GC $\alpha$ (Fig. 3D). Together, these results clearly identify GC $\alpha$ as a functional GC and establish that it generates the cGMP signal in asexual blood-stage schizonts essential for PKG activation, PKG-dependent $\mathrm{Ca}^{2+}$ release, and ultimately merozoite egress.

Chemical complementation of GC $\alpha$-null parasites with PET-cGMP rescues the egress defect. Since the egress phenotype observed in GC $\alpha$-deficient parasites is most likely due to their inability to produce cGMP to activate PKG, we reasoned that bypassing the need for cGMP synthesis by directly activating PKG could potentially rescue the egress defect. We also anticipated that this might reveal a second phenotype resulting from loss of the ATPase domain. To test this notion, we supplemented cultures containing highly mature RAP-treated cycle $0 \mathrm{GC} \alpha$ :HA:cKO schizonts with either cGMP or two cGMP analogues, 1- $\mathrm{NH}_{2}$-cGMP and PET-cGMP, both of which have previously been shown to activate recombinant apicomplexan PKG $(37,38)$. While cGMP and $1-\mathrm{NH}_{2}-$ cGMP show similar levels of membrane permeability, PET-cGMP is 50-fold more lipophilic (https://www.biolog.de/technical_info_lipophilicity-data) due to its possession of a $\beta$-phenyl-1, $\mathrm{N}^{2}$-ethenoguanosine extension to the purine ring of cGMP. After $1 \mathrm{~h}$ of incubation in the presence of various concentrations of $\mathrm{CGMP}, 1-\mathrm{NH}_{2}-\mathrm{CGMP}$, or PETcGMP, microscopic examination of the cultures revealed that PET-cGMP was highly effective at rescuing the egress block, resulting in the release of merozoites at all concentrations tested (Fig. 4A; see also Fig. S4A). While rings with normal morphology could be observed in the cultures containing the lower concentrations of PET-cGMP $(15,31.2$, and $62.5 \mu \mathrm{M})$, the cultures supplemented with higher PET-cGMP concentrations contained numerous intracellular or extracellular pyknotic forms, indicating toxicity (Fig. 4B). Few rings were observed in the cGMP and 1- $\mathrm{NH}_{2}-\mathrm{CGMP}$-treated cultures, indicating limited activity of these compounds (Fig. 4A). It was concluded that PETcGMP efficiently reversed the egress defect displayed by the GC $\alpha$-deficient parasites (Fig. 4A).

To determine the optimum concentrations of PET-cGMP to sustain replication of the $\mathrm{GC} \alpha$-null parasites, parasite proliferation was assessed over a $72-\mathrm{h}$ period (1.5 erythrocytic cycles) in the continuous presence of various concentrations of PET-cGMP. Interestingly, this revealed that none of the concentrations tested could rescue replication of the GC $\alpha$-null parasites (Fig. 4C), including those concentrations that efficiently rescued egress in the short-term assays described above. To try to understand this, we performed parallel assays assessing growth of control, DMSO-treated GC $\alpha$ :HA:cKO parasites in cultures supplemented with the same range of PET-cGMP concentrations. This revealed that PET-cGMP is toxic, with a half-maximal effective concentration $\left(\mathrm{EC}_{50}\right)$ of $12.49 \pm 0.06 \mu \mathrm{M}$ (Fig. 4C). Given its capacity to rescue the egress defect in GC $\alpha$-null parasites, we considered that the toxic effect of prolonged exposure to the compound is likely explained by premature activation of PKG as the parasites matured, perhaps resulting in premature egress and release of noninvasive merozoites, a phenomenon previously observed upon treatment of schizonts with zaprinast (31). Furthermore, since bioinformatic analysis has revealed that PKG is the only predicted cGMP effector in Plasmodium parasites, it is unlikely that the toxic effects observed following PETcGMP treatment are due to the activation of off-target effectors. In support of this model, further work showed that mature DMSO- and RAP-treated GC $\alpha$ :HA:cKO schizonts tolerated short-term incubation with $30 \mu \mathrm{M}$ PET-cGMP, followed by washing off the compound $\sim 10 \mathrm{~h}$ later, after the majority of schizonts had ruptured and formed rings; under these conditions, the new rings successfully matured to form cycle 1 schizonts (see Fig. S4B). Based on these findings, we used flow cytometry to quantify the degree of rescue that could be achieved over a single egress/invasion cycle. Quantification of cycle 1 parasite levels the following day showed that the presence of PET-cGMP during egress and invasion produced a 3.6-fold increase in parasitemia in the GC $\alpha$-null cultures, while parallel cultures of GC $\alpha$-null parasites lacking PET-cGMP completely failed to expand (Fig. 4D). In contrast, the replication rate of control 
A

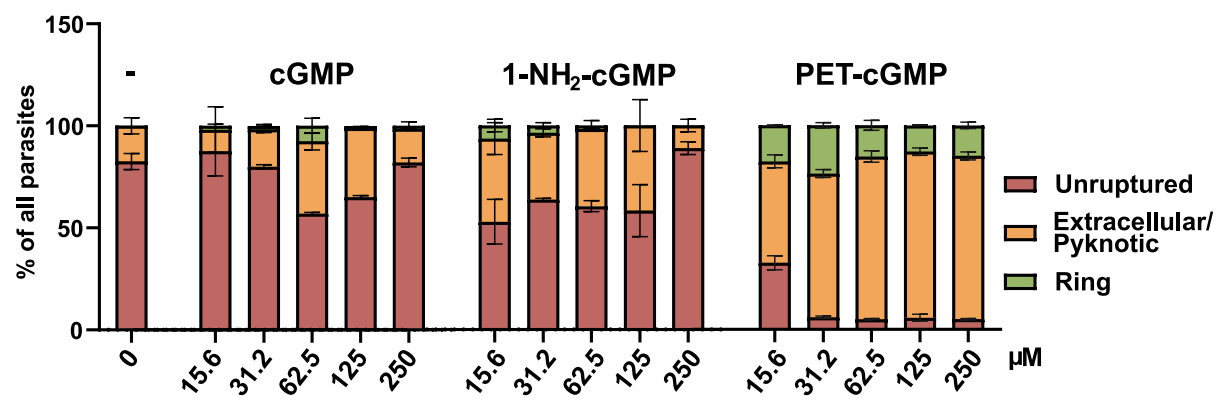

B

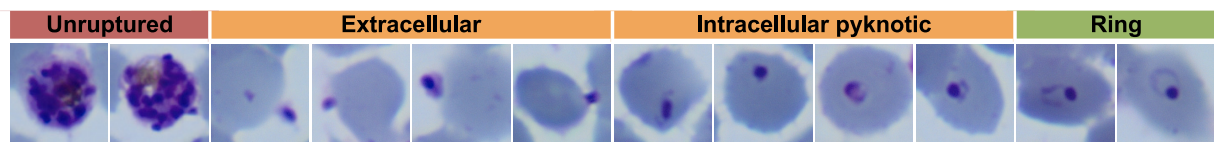

C

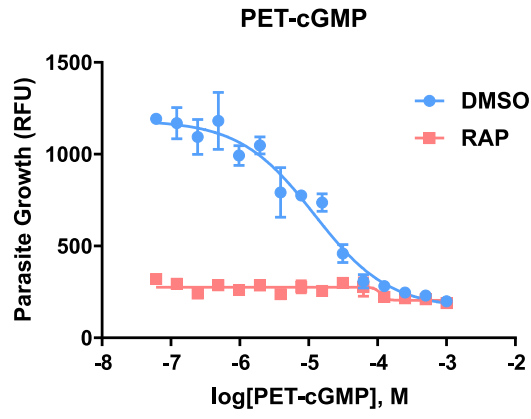

D

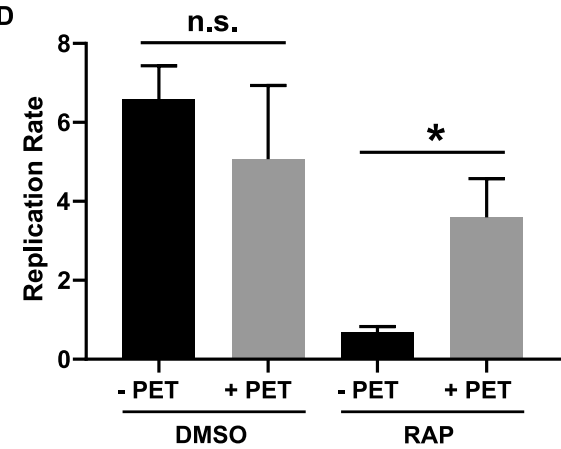

FIG 4 The addition of PET-cGMP efficiently rescues the egress block phenotype of GC $\alpha$ knockout schizonts. (A) Quantification of the effect of various concentrations of cGMP, 1- $\mathrm{NH}_{2}-\mathrm{CGMP}$, and PET-cGMP on the reversal of the egress block and promotion of ring-stage development in RAP-treated GC $\alpha$ :HA:cKO parasites. Ten microscopic fields per condition were counted blindly by two researchers, and parasites were scored as either unruptured (trophozoites and schizonts), uninvaded (free merozoites and pyknotic), or invaded (ring stages). The data are presented as proportions of the different forms of total parasites counted. Values are means of counts by each researcher. Error bars indicate the standard deviations. (B) Representative Giemsa images of pyknotic forms observed in smears taken from RAP-treated GC $\alpha$ :HA:cKO cultures $1 \mathrm{~h}$ after the addition of 62.5 $\mu \mathrm{M}$ PET-CGMP in the experiment quantified in panel A. (C) Continuous PET-cGMP treatment is toxic and does not rescue GC $\alpha$-null parasite growth. Growth of DMSO- and RAP-treated GC $\alpha$ :HA:cKO parasites at different concentrations of PET-cGMP as measured in a 72-h SYBR green growth assay. Assays were repeated twice, and the mean $\mathrm{EC}_{50}$ of PET-cGMP was measured as $12.49 \pm 0.06 \mu \mathrm{M}$ in wild-type parasites (DMSO). Error bars indicate the standard deviations. (D) Comparison of replication rates from cycle 0 to cycle 1 of DMSO- and RAP-treated $\mathrm{GC} \alpha$ :HA:cKO parasites cultured in the presence or absence of $30 \mu \mathrm{M}$ PET-cGMP, as measured by flow cytometry-based counting of SYBR green-positive cells. PET-CGMP was added when mature segmented schizonts appeared in culture and washed off $\sim 10 \mathrm{~h}$ later, once rings had formed. Data points plotted are means from two repeat experiments, each performed in triplicate. Error bars indicate the standard deviations. Statistical significance was measured by a ratio paired $t$ test, with " ${ }^{\prime *}$ signifying $P>0.05(0.0177)$. n.s., not significant.

parasites was slightly reduced (from 6.5- to 5 -fold) by similar short-term treatment with PET-cGMP, perhaps indicative of the low levels of toxicity of the compound (Fig. 4D).

These results confirmed the capacity of PET-CGMP to rescue the egress defect in the $\mathrm{GC} \alpha$-null parasites. Of particular significance, since the conditional strategy used to disrupt the GC $\alpha$ gene was designed to excise key segments of both the cyclase and the ATPase-encoding sequences, the results indicated either that the ATPase domain has no essential function or that its function is to stimulate or activate CGMP synthesis, allowing its role to be bypassed by the presence of the CGMP analogue.

A conserved catalytic Asp residue within the ATPase domain is required for parasite survival. The N-terminal portion of GC $\alpha$ encodes a putative P-type ATPase that shares closest homology with type IV ATPases (P4-ATPases) (5) which in other organisms flip phospholipids from the outer to the inner leaflet of a lipid bilayer (12, 13). P-type ATPases possess 10 transmembrane helices that facilitate transport of 
A

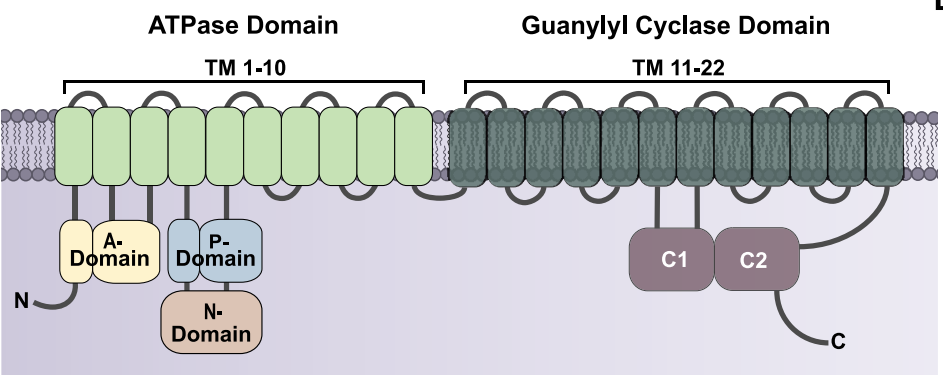

C

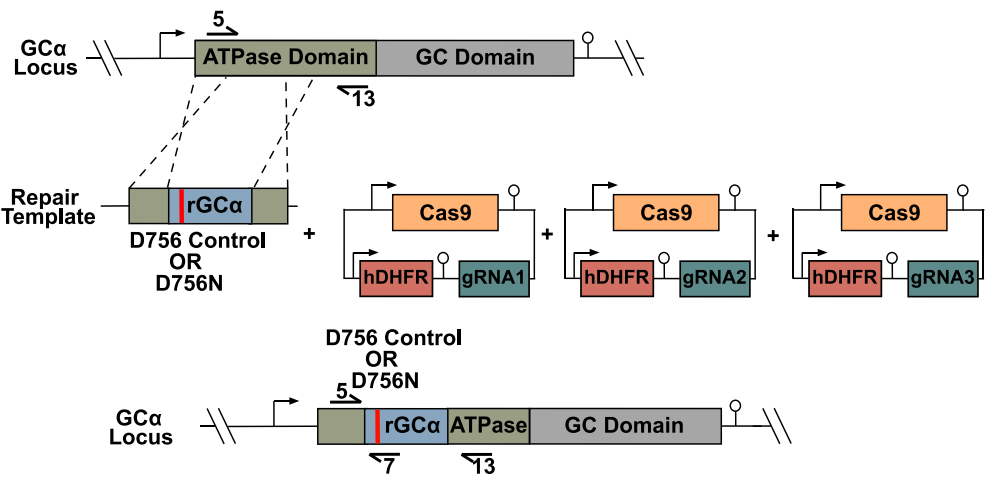

ATP8A1 LGQVKYIFSDKTGTLTCNVMQFKKC 424 Drs2p LGQIEYIFSDKTGTLTRNIMEFKSC 575 PfGC $\alpha$ LGNVDFIFTDKTGLTNNNMTENMC 771 TgGC LGQVDFIFTDKTGTITENDMTFSMC 797 ATP8B1 LGQIHYIFSDKTGTLTQNIMTFKKC 469 Dnf1 LGQVEYIFSDKTGTLTQNVMEFKKC 682 SERCA LGCTSVICSDKTGTLTTNQMSVCKM 366 $\mathrm{Na}+/ \mathrm{K}+$ LGSTSTICSDKTGTLTQNRMTVAHM 396

D

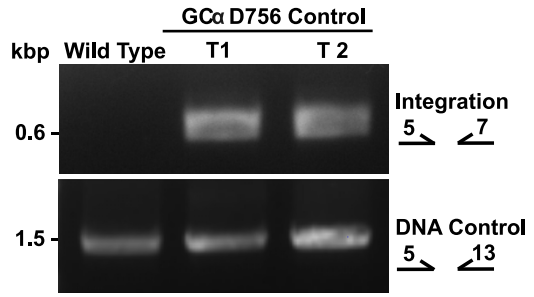

FIG 5 Key functional domains of the ATPase domain of GC $\alpha$ and strategy for substitution of the phosphorylation site aspartate. (A) Cartoon showing details of the predicted ATPase topology of $P$. falciparum GC $\alpha$. (B) Amino acid alignment of the phosphorylation site within the Pdomain of P4-ATPases. The aligned sequences are as follows: GC $\alpha$ (PlasmoDB identifier PF3D7_1138400), human ATP8A1 (Q9Y2Q0-2), human ATP8B1 (O43520), yeast Drs2p (P39524), yeast Dnf1 (P32660), T. gondii GC (EPT31724), P. falciparum GC $\beta$ (PlasmoDB identifier PF3D7_1360500), rabbit SERCA Ca ${ }^{2+}$-ATPase isoform 1a (P04191), and dogfish $\mathrm{Na}^{+} / \mathrm{K}^{+}$-ATPase (Q4H132). (C) Schematic representation of the marker-free CRISPR/ Cas9-mediated approach used to introduce synonymous or nonsynonymous mutations into the ATPase domain of GC $\alpha$ in the GC $\alpha$ :HA line. Arrows with numbers represent the relative position of oligonucleotide primers used for diagnostic PCR. A pool of three Cas9 plasmids harboring different gRNA cassettes was transfected, along with a linearized plasmid containing synonymous mutations (D756 control) or mutations resulting in an amino acid change (D756N), flanked by recodonized ( $\mathrm{rGC} \alpha$ ) and endogenous GC $\alpha$ sequences serving as the template for homology-directed repair. Promoters/5' UTRs and 3' UTRs/terminators are indicated by arrows and lollipops, respectively. (D) Diagnostic PCR evidencing modification of the GC $\alpha$ locus by integration of the D756 control construct in two independent transfections. The PCR primers used are indicated on the right, and their respective binding sites are shown in panel $\mathrm{C}$.

ligands according to the Post-Albers mechanism (39). This requires the following cytoplasmic components: a nucleotide binding domain ( $\mathrm{N}$-domain), which binds ATP; a phosphorylation domain (P-domain), which contains a highly conserved aspartate (Asp) residue that becomes autophosphorylated to form an aspartyl phosphate intermediate; and an actuator domain (A-domain), which dephosphorylates the phosphorylation domain $(40,41)$ (Fig. 5A). ATP-dependent autophosphorylation of the conserved Asp, which lies within a DKTGT motif, leads to a rotational change in the actuator domain surrounding the phosphorylation site; dephosphorylation is then coupled to inward transport of phospholipids or ions in the respective P-type ATPase families. Alignment of the GC $\alpha$ N-terminal region with other P4-ATPases, including human ATP8A1, for which a crystal structure has recently been determined (12), shows that of the 56 residues identical in P4-ATPases and P2-ATPases $\mathrm{Ca} \mathrm{Ca}^{2+}$ transporting SERCA and a $\mathrm{Na}^{+}-\mathrm{K}+\mathrm{P}$-type ATPase) that are required for ATP binding and catalysis, 47 are also identical in $\mathrm{GC} \alpha$ and 2 are similar, fully consistent with the $\mathrm{GC} \alpha$ sequence representing a functional ATPase domain (see Fig. S5).

Previous functional investigations (reviewed in reference 9) and two recent structural studies on yeast Drs $2 p$ and human ATPase8A1 in complex with their respective CDC50 partners $(12,13)$ have demonstrated the importance of the transmembrane domains of P4-ATPases, particularly TM1 to TM4 (but also TM6), for lipid binding and translocation. Key among these is the PISL motif in TM4 which is highly conserved in P4-ATPases and is also present in the GC $\alpha$ sequence. This motif constitutes an 
important difference between the phospholipid and cation transporting ATPases and is crucial for binding and translocation of their respective substrates. P4-ATPases have a PISL motif whereas the ion transporters have a PEGL motif. GC $\alpha$ has a PISI motif at this position which contains the crucial IS pair diagnostic of phospholipid binding/ translocation rather than cation binding/translocation (see Fig. S5). The QQ motif at the $\mathrm{C}$ terminus of TM1 contains noncharged polar residues (e.g., $\mathrm{Q}$ and $\mathrm{N}$ ) which in human ATP8A1 determine selectivity for PS in P4-ATPases. $\mathrm{Q}$ and $\mathrm{N}$ can hydrogen bond to the negative charge of the polar head group of PS, whereas the presence of small nonpolar residues ( $\mathrm{A}$ and $\mathrm{G}$ ) in place of the $\mathrm{QQ}$ motif preclude hydrogen bonding to PS and are features of P4-ATPases that translocate PC (12). GC $\alpha$ has a QN pair corresponding to the QQ motif of human ATP8A1, which is compatible with flipping of PS.

To address whether the ATPase domain of GC $\alpha$ is required for blood-stage replication, we investigated whether parasites could replicate following substitution of the conserved Asp (D756) in a manner designed to block formation of the aspartyl phosphate intermediate and thus ablate any enzymatic activity of the ATPase. To do this, we employed marker-free CRISPR/Cas9-mediated gene editing to introduce an asparagine (Asn) substitution of GC $\alpha$ D756 (Fig. 5B) (Fig. 5C; see also Fig. S1), since this substitution has been shown to completely ablate the activity of the yeast Drs $2 p$ P-Type ATPase and is expected to cause minimal structural changes to the ATPase (42). In parallel control manipulations, we used a similar approach to introduce synonymous mutations that did not alter the amino acid sequence but that could be differentiated from the wild-type locus at the nucleotide level. While parasites with a modified locus appeared in two of two independent transfections in these control cultures by 2 weeks posttransfection (Fig. 5D), no parasites emerged from three independent D756N transfections performed in parallel (one was performed in parallel with the successful integration of the loxPint) even following extended culture (6 weeks). Our failure to obtain viable parasites harboring the D756N mutation after three attempts, while readily obtaining transgenic parasites possessing synonymous mutations of the D756 codon, strongly suggests that an active ATPase domain is required for parasite survival. These data, combined with our ability to rescue the GC $\alpha$-null growth phenotype by chemical complementation with PET-cGMP, indicate that the ATPase domain acts upstream of and is required for GC activity and that the ultimate role of the ATPase domain is to facilitate cGMP synthesis.

\section{DISCUSSION}

Although the single GC in the related apicomplexan parasite $T$. gondii (14-17) and GC $\beta$ in $P$. falciparum (23), $P$. berghei $(24,25)$, and $P$. yoelii (8) have been previously analyzed by reverse genetics, there have as yet been no functional studies of GC $\alpha$ in the asexual blood stages of Plasmodium. Here, we have made important advances in understanding the essentiality and function of this key component of the cGMP signaling pathway.

Epitope tagging of the endogenous P. falciparum GC $\alpha$ locus indicated that the protein is located in puncta within the confines of individual merozoites within mature schizonts. We propose that these puncta represent cytoplasmic membranous structures, consistent with the architecture of the protein which has 22 predicted transmembrane helices. It is likely that the GC $\alpha$ topology is orientated such that the twin GC catalytic domains face the cytosol in order to allow synthesis of cGMP to activate PKG, the majority of which is cytosolic (43). Our observed localization for $P$. falciparum GC $\alpha$ is reminiscent of that recently described for GC $\alpha$ in $P$. yoelii gametocytes (44). In contrast, in Toxoplasma (14-17) the single GC has been localized to the plasma membrane, primarily at the apical pole, in extracellular parasites, a localization similar to that of GC $\beta$ in P. yoelii ookinetes. Therefore, unlike PyGC $\beta$ and TgGC, there is no evidence of subcellular partitioning of cGMP generated by PfGC $\alpha$ in asexual blood stages.

Epitope-tagged $P$. falciparum GC $\alpha$ migrated in Western blots as an $\sim 175$-kDa species, much smaller than the predicted full-length protein $(\sim 500 \mathrm{kDa})$. A similar 
phenomenon has been observed in P. yoelii, where GC $\alpha$ migrates at $\sim 175 \mathrm{kDa}$ instead of the predicted full-length $\sim 450 \mathrm{kDa}(44)$, as well as in $T$. gondii, where TgGC predominantly migrates at $\sim 125$ and $\sim 75 \mathrm{kDa}$, with only small amounts of the $\sim 460-\mathrm{kDa}$ species corresponding to full-length protein detected in some studies $(14,15,17,18)$. Given the evidence suggesting that the Toxoplasma GC needs to be full length to be functional (15), it seems that the 175-kDa species observed in our study likely represents an $\mathrm{N}$-terminally truncated proteolytic fragment, most likely resulting from protein instability during detergent extraction and that full-length $P$. falciparum GC $\alpha$ protein is present but below detection limits in our experiments.

Conditional deletion of a large segment of GC $\alpha$ spanning both the GC catalytic domain and the ATPase-like domain prevented asexual blood-stage replication, with a selective block in egress. This finding was in contrast to results generated in a previous global transposon-based gene knockout study which suggested that GC $\alpha$ was dispensable for blood-stage replication (45). However, that study acknowledged that false negatives can be obtained using their approach depending upon the position of transposon insertion. In an in vivo global gene knockout study in $P$. berghei, parasites in which GC $\alpha$ was disrupted grew slowly and were close to the cutoff for those essential for blood-stage replication (46), which is more consistent with our findings in $P$. falciparum.

We have previously reported that GC $\alpha$ possesses all the conserved amino acid residues required for catalytic activity (6). However, the ability of GC $\alpha$ to synthesize cGMP had previously not been demonstrated. The lack of detectable cGMP generation in $\mathrm{GC} \alpha$-null parasites confirms that it is a functional GC and also indicates that $\mathrm{GC} \beta$, which is expressed only during mosquito stage development, cannot compensate for the absence of GC $\alpha$ in blood stages. The lack of cGMP synthesis fully explains the egress phenotype of GC $\alpha$-null parasites, because activation of PKG by cGMP is known to be essential for egress $(30,31)$. PKG activity also induces calcium release from internal stores in $P$. falciparum schizonts, which is required for egress. This is concordant with our finding that GC $\alpha$-null schizonts did not mobilize calcium in response to cGMP elevation by the PDE inhibitor zaprinast. Together, these results establish that GC $\alpha$ is the key regulator upstream of PKG activity in $P$. falciparum blood stages. Disruption of the Toxoplasma GC also abolishes the rises in cytosolic $\mathrm{Ca}^{2+}$ required for secretion of micronemal proteins $(17)$ and motility $(47,48)$. Recent work in Toxoplasma has revealed a feedback loop between CGMP and CAMP signaling (18). However, unlike in Toxoplasma, where CAMP signaling is a negative regulator of egress, $P$. falciparum merozoite egress is not affected following disruption of either AC $\beta$ or PKA (49). Inhibition of soluble guanylyl cyclase activity following phosphorylation by PKG has been reported in other systems (50), and we have previously identified that PfGC $\alpha$ is phosphorylated at two independent sites in a PKG-dependent manner (33), pointing toward a possible feedback loop whereby activated PKG may regulate GC $\alpha$. Future work will be required to determine the significance of these phosphosites.

It has been suggested that the chemical gradient of phospholipids generated by P4-ATPases is akin to the chemical gradient of ions created by P2-ATPases to mediate signal transduction (51). To investigate whether the ATPase-like domain in PfGC $\alpha$ is likely to be a catalytically active ATPase, we attempted to generate parasites possessing a substitution of a highly conserved aspartate (D756) that is autophosphorylated in functional ATPases. These parasites could not be selected, while those engineered to reconstitute the wild-type aspartate proliferated normally, providing evidence that the ATPase domain is catalytically active and serves an essential function. Our finding that the GC $\alpha$-null phenotype could be rescued by addition of the membrane-permeable cGMP analogue PET-cGMP, strongly suggests that the function of the ATPase domain is upstream of and directly related to CGMP synthesis. Our findings are consistent with previous studies in Toxoplasma where the ATPase domain was also shown to be critical for GC function $(14,15,17)$. Complementation of GC-null Toxoplasma with a panel of mutants demonstrated that the ATPase domain is required for trafficking of GC, activity 
and maximal GC function since stimulated micronemal secretion was only partially reduced in the ATPase D728A mutant, while natural microneme secretion was prevented (15). Although our data suggest that the activity of the ATPase domain of GC $\alpha$ is an essential upstream regulatory factor for CGMP synthesis, our study does not further address its biochemical activity or indeed how it facilitates CGMP synthesis. The single Toxoplasma GC is involved in sensing phosphatidic acid, as well as changes in $\mathrm{pH}$ and potassium levels, to mediate egress $(14,17)$. A recent suggestion that $P$. falciparum GC $\alpha$ might flip phosphatidylcholine (52) to mediate cGMP-stimulated egress was not supported by direct evidence of GC $\alpha$-mediated flipping of phosphatidylcholine. Our analysis has shown the presence of a QN pair in $P$. falciparum at the $C$ terminus of TM1 in GC $\alpha$, which corresponds to the QQ motif of human ATP8A1 (12), suggesting that the sequence is compatible with flipping of PS. Future work will be needed to determine whether regulation of CGMP synthesis by GC $\alpha$ requires flipping or sensing of phospholipids by the ATPase domain and whether generation of lipid asymmetry across membranes contributes to activation of cGMP synthesis. This could be achieved by complementation of our conditional knockout line with various versions of GC $\alpha$ harboring the mutations in the ATPase domain.

Synthesis of cGMP by GC $\alpha$ has been linked to the stimulation of gametogenesis by xanthurenic acid (XA) (20), but it is not clear whether XA stimulates GC $\alpha$ activity directly or indirectly through other protein mediators. However, a study in $P$. yoelii has recently identified a protein called GEP1 that interacts with $\mathrm{GC} \alpha$, showing that both are required for XA-stimulated gametogenesis (44). Although cGMP synthesis and PKG activation are required for merozoite egress, the nature of the upstream signal that activates $\mathrm{GC} \alpha$ in Plasmodium blood stages is unknown. Just prior to natural egress in Toxoplasma, the parasitophorous vacuole is acidified which triggers micronemal secretion (17). A similar mechanism may operate in Plasmodium. Future work will be needed to determine the events that occur upstream of cGMP signaling in blood stages and to understand the role of the P4-type ATPase domain in mediating the egress signal.

\section{MATERIALS AND METHODS}

Small molecules and antibodies. WR99210 was kindly provided by Jacobus Pharmaceuticals (Plainsborough Township, NJ). The PKG inhibitor compound 2 (4-[7-[(dimethylamino)methyl]-2-(4-fluorphenyl)imidazo[1,2- $\alpha$ ]pyridine-3-yl]pyrimidin-2-amine) was synthesized by LifeArc (London, United Kingdom). Rapamycin, calcium ionophore A23187, the cysteine protease inhibitor E64, and the PDE inhibitor zaprinast were all purchased from Sigma-Aldrich (St. Louis, MO). The calcium chelator Fluo4-AM was purchased from Thermo Fisher Scientific (Waltham, MA). cGMP, PET-cGMP, and 1- $\mathrm{NH}_{2}-$ cGMP were purchased from the Biolog Life Science Institute (Bremen, Germany).

Rat monoclonal anti-HA tag antibody (clone 3F10) was purchased from Roche LifeScience (Penzberg, Germany) and rabbit anti-human PKG antibody from Enzo Life Sciences (Farmingdale, NY). A rabbit polyclonal antibody against MSP1-30 (53) as well as a rabbit anti-AMA1 antibody raised against the ectodomain (54) and rabbit anti-SERA5 (55) were all described previously. A mouse monoclonal antibody to plasmepsin $\mathrm{V}$ was kindly provided by Daniel Goldberg (Washington University School of Medicine in St. Louis, MO).

P. falciparum culture and synchronization. P. falciparum asexual blood stages were cultured in human erythrocytes (National Blood Transfusion Service, London, United Kingdom) and complete medium (CM) consisting of RPMI 1640 medium (Life Technologies, CA) supplemented with $0.5 \%$ AlbuMAX type II (Gibco), $50 \mu \mathrm{M}$ hypoxanthine, and $2 \mathrm{mM}$ L-glutamine. Parasite cultures were incubated at $37^{\circ} \mathrm{C}$ and gassed with $90 \% \mathrm{~N}_{2}, 5 \% \mathrm{CO}_{2}$, and $5 \% \mathrm{O}_{2}$ according to standard procedures (56). Parasitemias were routinely monitored by examination of thin blood films fixed with $100 \%$ methanol and stained with $10 \%$ Giemsa stain in phosphate buffer ( $8 \mathrm{mM} \mathrm{KH}_{2} \mathrm{PO}_{4}, 6 \mathrm{mM} \mathrm{Na}_{2} \mathrm{HPO}_{4}[\mathrm{pH} 7.0]$ ).

Tightly synchronous parasites were obtained by purifying segmented schizonts on a $70 \%$ isotonic Percoll (GE Healthcare, Arlington Heights, IL) cushion and allowing them to invade fresh erythrocytes for 1 to $2 \mathrm{~h}$ while shaking. Unruptured schizonts were lysed by treating with $5 \% \mathrm{D}$-sorbitol (Sigma) for 10 $\min (57)$ to obtain highly pure and synchronous ring-stage cultures.

Induction of DiCre activity was achieved by treating early ring-stage parasites ( 2 to $10 \mathrm{~h}$ postinvasion) with $50 \mathrm{nM}$ RAP for 2 to $3 \mathrm{~h}$. Control parasites were treated with an equivalent volume of the vehicle DMSO (0.5\% [vol/vol]).

Transfection of $\boldsymbol{P}$. falciparum schizonts. Highly synchronous late-stage schizonts were used for transfection as previously described (35) using an Amaxa 4D-Nucleofector system (Lonza, Basel, Switzerland). For each transfection, $\sim 1.25 \times 10^{8}$ Percoll-enriched schizonts were resuspended in supplemented P3 primary cell solution containing 20 to $50 \mu \mathrm{g}$ of plasmid DNA and transferred to a Nucleocuvette. Parasites were electroporated using the FP158 setting and then transferred back into 
culture. Modified parasites were selected $24 \mathrm{~h}$ posttransfection by addition of WR99210 (2.5 nM). WR99210 was removed 7 days later when selecting for the presence Cas9/gRNA plasmids (GC $\alpha$ :HA:cKO and ATPase mutation) or left on until parasites emerged and then cycled on/off WR (GC $\alpha$ :HA).

Plasmid construction. Primers used throughout this study were ordered from Integrated DNA Technologies (IDT, Coralville, IA) and are listed in Table S1 in the supplemental material. To generate the GC $\alpha$ :HA parasite line, a 1.9-kb fragment corresponding to the $3^{\prime}$ end of the GC $\alpha$ coding region was PCR amplified with primers 14 and 15 and cloned into pHH1_PreDiCre_A_deltaH_deltaE (35) via EcoRV and Xhol restriction sites, upstream of the sequence encoding a triple hemagglutinin $(3 \times \mathrm{HA})$ tag. The resulting plasmid pHH1_PreDiCre_GC $\alpha-3 \times \mathrm{HA}$ was transfected into the 3D7/1G5DiCre line constitutively expressing dimerizable Cre recombinase (35). Transfected cultures were selected with WR99210 and then subjected to drug cycling to enrich for parasites having integrated the plasmid via single crossover recombination. Cultures were finally treated with RAP to activate Cre recombinase to recycle the hdhfr resistance marker. A clonal GC $\alpha$ :HA line sensitive to WR99210 was selected and further modified by CRISPR/Cas9-mediated gene editing to introduce a loxPint sequence into the ATPase domain of GC $\alpha$ to generate the GC $\alpha$ :HA:CKO line. A pUC19-based repair template was generated by first amplifying a 545bp $5^{\prime}$ homology region and a 627-bp 3' homology region from genomic DNA using the primer pairs 16/ 17 and 18/13, respectively. The two PCR products were fused by overlap extension PCR using primers $16 / 13$ and InFusion cloned into the HindIII and EcoRI sites of pUC19. A synthetic recodonized region of the GC $\alpha$ gene from bp 2109 to bp 2340 containing a SERA2-derived loxPint was ordered as a gBlock (IDT) and InFusion cloned into the Afll and BamHI sites located between the 5' and 3' homology regions. The repair template was linearized using Pvul and transfected, along with three pooled pDC2 plasmids, each encoding the Cas9 protein, the hDHFR selection cassette (which confers resistance to the antifolate WR99210), and a unique sgRNA sequence targeting either TTTAATATGTGTTCTATAGC, TCTATAGCAGGAAAAACATA, or CATATTCATCATAATCATTT. To mutate the ATPase domain of GC $\alpha$, the repair template used to introduce the loxPint was modified to introduce either a D756N mutation, which would block formation of the aspartyl phosphate intermediate, or a wild-type D756 synonymous mutation, which would serve as a control. The repair templates were generated by replacing the loxPint flanked by the Bglll and Kpnl sites with overlap extension PCR products from primer sets 19/20 and $21 / 22$ to introduce the D756N and D756 alleles, respectively. Each repair template plasmid was linearized with Pvul, combined with the pool of three pDC2 Cas9 plasmids mentioned above, and transfected into wild-type 3D7 parasites.

Limiting dilution to generate clonal parasite lines. Clonal parasite lines were obtained by limiting dilution combined with a plaque formation readout as previously described (58). The hematocrit and parasitemia of parasite cultures were determined by using a hemocytometer and by counting Giemsastained thin blood smears. Briefly, parasite cultures were diluted to give 0.3 parasites in $200 \mu$ l of culture at $1 \%$ hematocrit per well in a 96-well flat-bottom plate. After 9 days, plaque formation was assessed by using an EVOS FL cell imaging system (Thermo Fisher Scientific). Wells containing single plaques were subsequently expanded and analyzed by PCR.

Diagnostic PCRs. Integration of the $3 \times \mathrm{HA}$-tagging construct into the GC $\alpha$ locus was confirmed using primers 1/2. RAP-induced excision of the hDHFR cassette to create the GC $\alpha$ :HA line was validated using primers 1/4. Integration of the artificial loxPint into the ATPase domain of the GC $\alpha$ locus to generate the GC:HA:cKO line was confirmed using primers 5/7, while primers 5/6 were used to detect the presence of wild-type locus. Cre-mediated excision was validated using the primer pairs 10/11 and 12/11 to detect the unexcised and excised loci, respectively. Primers $8 / 9$, which amplify a segment in an unmodified distal locus, served as a DNA quality control.

SYBR green growth inhibition assays. To determine the effect of various test compounds on parasite growth, their $\mathrm{EC}_{50} \mathrm{~s}$ were determined by using the SYBR green growth inhibition assay adapted from a previous study (59). Test compounds were added as a series of 2-fold serial dilutions in triplicate to 96well flat-bottom plates. Wells containing no drug or $10 \mathrm{nM}$ chloroquine were also included in each plate and served as negative and positive controls, respectively. Synchronous ring-stage parasites were added to achieve a starting parasitemia of $2 \%$ at $1 \%$ hematocrit and incubated at $37^{\circ} \mathrm{C}$ in a sealed gassed box for $72 \mathrm{~h}$. Parasites were then lysed in buffer containing $20 \mathrm{mM}$ Tris, $5 \mathrm{mM}$ EDTA, 0.008\% saponin, 0.08\% Triton X-100, and $1 \times$ SYBR green I (Molecular Probes, Eugene, OR) at pH 7.5 and incubated for $1 \mathrm{~h}$ at room temperature. SYBR green fluorescence was measured using a SpectraMax M3 plate reader (Molecular Devices, San Jose, CA) with excitation and emission wavelengths of 485 and $535 \mathrm{~nm}$, respectively. $\mathrm{EC}_{50}$ values were determined by nonlinear regression analysis.

Fluorescence-activated cell sorting analysis to measure parasite growth and DNA content. Parasite cultures were seeded in triplicate wells per condition and samples were fixed in $4 \%$ formaldehye, $0.1 \%$ glutaraldehyde in PBS containing $1 \times$ SYBR green I (Molecular Probes) and stored at $4^{\circ} \mathrm{C}$ overnight. The fixative was aspirated, and the cells were washed in PBS and then analyzed using a BD LSR II flow cytometer (BD Biosciences, Franklin Lakes, NJ), with 50,000 events collected for each sample. FlowJo 7 analysis software (FlowJo LLC, Ashland, OR) was used to analyze the data.

Immunofluorescence microscopy. Air-dried thin blood smears were fixed in $4 \%$ formaldehyde in phosphate-buffered saline (PBS) for $20 \mathrm{~min}$ at room temperature, followed by permeabilization with $0.1 \%$ Triton X-100 in PBS. Blocking and antibody binding steps were performed in PBS containing $3 \%$ bovine serum albumin. Dual staining experiments were performed sequentially, starting with rat anti-HA, to eliminate cross-reactivity of the anti-rat secondary antibody with mouse or rabbit IgG. The secondary antibodies used were anti-rat IgG antibody conjugated to Alexa Fluor 488, anti-rabbit IgG conjugated to Alexa Fluor 594, and anti-mouse IgG conjugated to Alexa Fluor 594, all highly cross-adsorbed. Slides were mounted in ProLong Gold Antifade Mountant containing 4',6'-diamidino-2-phenylindole (DAPI; 
Thermo Fisher Scientific). Images were acquired at $\times 100$ magnification using a Nikon Eclipse Ti fluorescence microscope fitted with a Hamamatsu C11440 digital camera and overlaid in ICY bioimage analysis software (icy.bioimageanalysis.org).

Time-lapse video microscopy. Parasite egress was monitored by differential interference contrast (DIC) coupled with fluorescence microscopy using a Nikon Eclipse Ti fluorescence microscope with a $60 \times$ oil immersion objective and fitted with a Hamamatsu C11440 digital camera. Segmented schizonts treated with C2 $(1.5 \mu \mathrm{M})$ overnight were Percoll enriched and resuspended in warm complete medium at $0.4 \%$ hematocrit, transferred to prewarmed Poly-L-Lysine $\mu$-Slide VI 0.4 (IBIDI, Planegg, Germany) imaging chambers and imaged on a temperature-controlled microscope stage held at $37^{\circ} \mathrm{C}$. To visualize DMSO- and RAP-treated parasites simultaneously, one culture was stained with $1 \mu \mathrm{g} / \mathrm{ml}$ Hoechst 33342 prior to washing off C2 and pooling the cultures, as previously described (60). Images were taken every $5 \mathrm{~s}$ for a total of 20 to $30 \mathrm{~min}$, and the resulting videos were processed and analyzed in ICY bioimage analysis software (icy.bioimageanalysis.org).

Microscopy of Giemsa-stained blood films. Thin blood films fixed with $100 \%$ methanol and stained with $10 \%$ Giemsa stain in phosphate buffer $\left(8 \mathrm{mM} \mathrm{KH}_{2} \mathrm{PO}_{4}, 6 \mathrm{mM} \mathrm{Na}_{2} \mathrm{HPO}_{4}[\mathrm{pH} 7.0]\right)$ were imaged using an Olympus BX51 microscope fitted with an Olympus SC30 digital color camera through a 100× oil immersion objective. Images were processed in Graphic (Picta, Inc.).

Parasite protein extraction, SDS-PAGE, and immunoblotting. Saponin-released parasites were lysed in 4 pellet volumes of ColP buffer (150 mM NaCl, 0.5 mM EDTA, 1\% NP-40, 10 mM Tris [pH 7.5]) supplemented with cOmplete EDTA-free protease inhibitor (Roche, Basel, Switzerland). Samples were incubated on ice for $10 \mathrm{~min}$ and centrifuged at $12,000 \times \mathrm{g}$ for $10 \mathrm{~min}$ at $4^{\circ} \mathrm{C}$, and the supernatant was collected. Reducing SDS sample buffer was added, and proteins were resolved on 4 to $15 \%$ Mini-Protean TGX stain-free precast gels (Bio-Rad, Hercules, CA) or 3 to 8\% NuPAGE Tris-acetate protein gels (Thermo Fisher Scientific) for high-molecular-weight proteins. Proteins were transferred onto nitrocellulose membranes using a semidry Trans-Blot Turbo transfer system (Bio-Rad) and blocked using 10\% skimmed milk in PBS containing $0.1 \%$ Tween 20 (PBST). Antibody incubations were carried out in $1 \%$ skimmed milk in PBST and washed in PBST. After incubation with secondary antibodies conjugated to near-infrared dyes, washed membranes were dried between Whatman 3MM blotting papers and imaged using an Azure c600 imaging system (Azure Biosystems, Dublin, CA) or a ChemiDoc imaging system (Bio-Rad).

Egress assays. Highly synchronous mature segmented schizonts from DMSO- and RAP-treated GC $\alpha$ : HA:cKO cultures that were treated with $\mathrm{C} 2(1.5 \mu \mathrm{M})$ at the early trophozoite stage were enriched on a Percoll gradient and washed several times in prewarmed RPMI. Parasites were resuspended in RPMI at $3.25 \times 10^{8}$ parasites $/ \mathrm{ml}$, and $65-\mu \mathrm{l}$ aliquots were dispensed into Eppendorf tubes. To harvest samples at each time point, parasites were pelleted at 9,000 $\times g$, and culture supernatants were purified using $0.22-$ $\mu \mathrm{m}$ Costar Spin-X centrifuge filters (Corning, Corning, NY). The parasite pellets from the first time point were retained as a parasite loading control. Samples were subjected to Western blot analysis and probed for SERA5 as a measure of merozoite egress.

Calcium release assays. Mature segmented schizonts from RAP- and DMSO-treated cultures were Percoll enriched, and $\sim 1.25 \times 10^{8}$ cells from each condition were incubated in phenol red-free RPMI containing $10 \mu \mathrm{M}$ Fluo-4-AM (Invitrogen, Carlsbad, CA) in the dark at $37^{\circ} \mathrm{C}$ for $45 \mathrm{~min}$. The parasites were washed twice in prewarmed phenol red-free RPMI and then incubated for 20 min to allow for de-esterification of the AM ester. The parasites were washed twice and resuspended in phenol red-free RPMI at $1.25 \times 10^{8}$ parasites $/ \mathrm{ml}$. Next, $100 \mu \mathrm{l}$ of resuspended parasites was added again to wells on the bottom half of a 96-well plate. Three wells containing phenol red-free RPMI were also included as a control. Baseline Fluo-4 fluorescence in each well was read at 22-s intervals for 3 min using a SpectraMax M3 plate reader (Molecular Devices) prewarmed to $37^{\circ} \mathrm{C}$ with excitation and emission wavelengths of 483 and $525 \mathrm{~nm}$, respectively. The plate was removed from the reader onto a heat block prewarmed to $37^{\circ} \mathrm{C}$, and the parasites were resuspended and transferred to wells containing test compounds to give the desired final concentrations of zaprinast $(75 \mu \mathrm{M})$, ionophore A23187 (20 $\mu \mathrm{M})$, and DMSO (1.5\%). The plate was placed back in the reader and read for a further $5 \mathrm{~min}$ at 22 -s intervals. All samples were run in triplicate. The relative fluorescence units from reads at each time point and condition were averaged, and baseline and DMSO control values were subtracted.

Measurement of intracellular cyclic nucleotide levels. Intracellular cyclic nucleotide levels in mature segmented schizonts were measured using enzyme-linked immunosorbent assay (ELISA)-based high-sensitivity direct CAMP and CGMP colorimetric assay kits (Enzo Life Sciences). Around $1.25 \times 10^{8}$ Percoll-purified schizonts were obtained from RAP- and DMSO-treated cultures to which C2 (1.5 $\mu \mathrm{M})$ had been added to prevent schizont rupture. The purified schizonts were incubated for 3 min in RPMI containing $\mathrm{C} 2$ only or $\mathrm{C} 2$ in the presence of the PDE inhibitor zaprinast (75 $\mu \mathrm{M})$. Parasites were then pelleted at $9,000 \times g$, resuspended in $100 \mu \mathrm{l}$ of $0.1 \mathrm{M} \mathrm{HCl}$, and incubated for $10 \mathrm{~min}$ at room temperature with intermittent vortexing to complete cell lysis. The samples were pelleted at $9,000 \times g$, and the supernatant was collected and stored at $-80^{\circ} \mathrm{C}$ until required. Once all biological replicates were collected, each sample was diluted by adding $400 \mu \mathrm{l}$ of $0.1 \mathrm{M} \mathrm{HCl}$. Samples and standards were acetylated in order to improve sensitivity according to the manufacturer's instructions.

The detection ranges were 0.078 to $20 \mathrm{pmol} / \mathrm{ml}$ and 0.08 to $50 \mathrm{pmol} / \mathrm{ml}$ for the cAMP and cGMP assays, respectively. All samples and standards were set up in duplicate. Absorbance was measured at $405 \mathrm{~nm}$ using a SpectraMax M3 plate reader (Molecular Devices).

Treatment of parasite cultures with cGMP analogues to rescue $\mathbf{G C} \alpha$ KO phenotype. Highly synchronous mature segmented schizonts from RAP-treated cultures at $\sim 48 \mathrm{~h}$ postinvasion were treated with CGMP, 1-NH $-\mathrm{NH}_{2}-\mathrm{CGMP}$, or PET-cGMP at concentrations ranging from 15.6 to $250 \mu \mathrm{M}$. Giemsa-stained thin blood smears were taken after $2 \mathrm{~h}$, and parasites were scored for their viability based on 
morphology. Parasites in 10 microscopic fields per condition were assigned to schizont, merozoite/ pyknotic intracellular, or ring-stage categories. Counts were performed blindly by two researchers. To measure the effect of $30 \mu \mathrm{M}$ PET cGMP on wild-type and GC $\alpha$ KO replication rates, the cGMP analogue was added to synchronous DMSO- and RAP-treated GC $\alpha$ :HA:cKO cultures when segmented schizonts appeared and washed off $10 \mathrm{~h}$ later; the parasites were then harvested for fluorescence-activated cell sorting (FACS) analysis at the ensuing early schizont stage.

Sequence alignments. Sequence alignments were performed using Clustal Omega, modified manually, and guided by the alignment presented in a recent P4-ATPase structural study (12).

Data analysis and statistical significance tests. GraphPad Prism 7 was used for all statistical analyses. The numbers of biological and technical replicates for each experiment are noted in the figure legends.

\section{SUPPLEMENTAL MATERIAL}

Supplemental material is available online only.

FIG S1, PDF file, $0.5 \mathrm{MB}$.

FIG S2, PDF file, 1.9 MB.

FIG S3, PDF file, $0.1 \mathrm{MB}$.

FIG S4, PDF file, 0.7 MB.

FIG S5, PDF file, 1.1 MB.

TABLE S1, PDF file, $0.10 \mathrm{MB}$.

\section{ACKNOWLEDGMENTS}

We thank Oriana Kreutzfeld for a contribution to plasmid construct generation. We also thank Daniel Goldberg for kindly providing the plasmepsin V antibody.

We are grateful for the BBSRC Ph.D. studentship awarded to S.D.N. (BB/M009513/1). We are also grateful to the Wellcome Trust for funding through a joint Senior Investigator Award to D.A.B. (106240/Z/14/Z) and M.J.B. (106239/Z/14/A) and Wellcome ISSF2 funding to the London School of Hygiene and Tropical Medicine. This work was also supported by funding to M.J.B. from the Francis Crick Institute, which receives its core funding from Cancer Research UK (FC001043), the UK Medical Research Council (FC001043), and the Wellcome Trust (FC001043).

We declare that there are no competing interests.

\section{REFERENCES}

1. Park M, Sandner P, Krieg T. 2018. cGMP at the centre of attention: emerging strategies for activating the cardioprotective PKG pathway. Basic Res Cardiol 113:24. https://doi.org/10.1007/s00395-018-0679-9.

2. Michalakis S, Becirovic E, Biel M. 2018. Retinal cyclic nucleotide-gated channels: from pathophysiology to therapy. Int J Mol Sci 19:749. https:// doi.org/10.3390/ijms19030749.

3. Baker DA, Kelly JM. 2004. Structure, function, and evolution of microbial adenylyl and guanylyl cyclases. Mol Microbiol 52:1229-1242. https://doi .org/10.1111/j.1365-2958.2004.04067.x.

4. Gardner MJ, Hall N, Fung E, White O, Berriman M, Hyman RW, Carlton JM, Pain A, Nelson KE, Bowman S, Paulsen IT, James K, Eisen JA, Rutherford K, Salzberg SL, Craig A, Kyes S, Chan M-S, Nene V, Shallom SJ, Suh B, Peterson J, Angiuoli S, Pertea M, Allen J, Selengut J, Haft D, Mather MW, Vaidya AB, Martin DMA, Fairlamb AH, Fraunholz MJ, Roos DS, Ralph SA, McFadden Gl, Cummings LM, Subramanian GM, Mungall C, Venter JC, Carucci DJ, Hoffman SL, Newbold C, Davis RW, Fraser CM, Barrell B. 2002. Genome sequence of the human malaria parasite Plasmodium falciparum. Nature 419:498-511. https://doi.org/10.1038/nature01097.

5. Baker DA, Drought LG, Flueck C, Nofal SD, Patel A, Penzo M, Walker EM. 2017. Cyclic nucleotide signalling in malaria parasites. Open Biol 7:170273.

6. Carucci DJ, Witney AA, Muhia DK, Warhurst DC, Schaap P, Meima M, Li JL, Taylor MC, Kelly JM, Baker DA. 2000. Guanylyl cyclase activity associated with putative bifunctional integral membrane proteins in Plasmodium falciparum. J Biol Chem 275:22147-22156. https://doi.org/10.1074/jbc M001021200.

7. Linder JU, Engel P, Reimer A, Krüger T, Plattner H, Schultz A, Schultz JE. 1999. Guanylyl cyclases with the topology of mammalian adenylyl cyclases and an N-terminal P-type ATPase-like domain in Paramecium,
Tetrahymena, and Plasmodium. EMBO J 18:4222-4232. https://doi.org/10 .1093/emboj/18.15.4222.

8. Gao H, Yang Z, Wang X, Qian P, Hong R, Chen X, Su X-Z, Cui H, Yuan J. 2018. ISP1-anchored polarization of $\mathrm{GC} \beta / \mathrm{CDC} 50 \mathrm{~A}$ complex initiates malaria ookinete gliding motility. Curr Biol 28:2763-2776 e6. https:// doi.org/10.1016/j.cub.2018.06.069.

9. Best JT, Xu P, Graham TR. 2019. Phospholipid flippases in membrane remodeling and transport carrier biogenesis. Curr Opin Cell Biol 59:8-15. https://doi.org/10.1016/j.ceb.2019.02.004.

10. Lopez-Marques RL, Holthuis JC, Pomorski TG. 2011. Pumping lipids with P4-ATPases. Biol Chem 392:67-76.

11. Poulsen LR, Lopez-Marques RL, Palmgren MG. 2008. Flippases: still more questions than answers. Cell Mol Life Sci 65:3119-3125. https://doi.org/ 10.1007/s00018-008-8341-6.

12. Hiraizumi M, Yamashita K, Nishizawa T, Nureki O. 2019. Cryo-EM structures capture the transport cycle of the P4-ATPase flippase. Science 365:1149-1155. https://doi.org/10.1126/science.aay3353.

13. Timcenko M, Lyons JA, Januliene $D$, Ulstrup JJ, Dieudonné $T$, Montigny $C$, Ash M-R, Karlsen JL, Boesen T, Kühlbrandt W, Lenoir G, Moeller A, Nissen P. 2019. Structure and autoregulation of a P4-ATPase lipid flippase. Nature 571:366-370. https://doi.org/10.1038/s41586-019-1344-7.

14. Bisio H, Lunghi M, Brochet M, Soldati-Favre D. 2019. Phosphatidic acid governs natural egress in Toxoplasma gondii via a guanylate cyclase receptor platform. Nat Microbiol 4:420-428. https://doi.org/10.1038/s41564 -018-0339-8.

15. Brown KM, Sibley LD. 2018. Essential cGMP signaling in toxoplasma is initiated by a hybrid P-type ATPase-guanylate cyclase. Cell Host Microbe 24:804-816. https://doi.org/10.1016/j.chom.2018.10.015.

16. Gunay-Esiyok O, Scheib U, Noll M, Guupta N. 2019. An unusual and vital protein with guanylate cyclase and P4-ATPase domains in a pathogenic 
protist. Life Sci Alliance 2:e201900402. https://doi.org/10.26508/lsa 201900402

17. Yang L, Uboldi AD, Seizova S, Wilde M-L, Coffey MJ, Katris NJ, YamaryoBotté Y, Kocan M, Bathgate RAD, Stewart RJ, McConville MJ, Thompson PE, Botté CY, Tonkin CJ. 2019. An apically located hybrid guanylate cyclase-ATPase is critical for the initiation of $\mathrm{Ca}^{2+}$ signaling and motility in Toxoplasma gondii. J Biol Chem 294:8959-8972. https://doi.org/10.1074/ jbc.RA118.005491.

18. Jia Y, Marq J-B, Bisio H, Jacot D, Mueller C, Yu L, Choudhary J, Brochet M, Soldati-Favre D. 2017. Crosstalk between PKA and PKG controls pH-dependent host cell egress of Toxoplasma gondii. EMBO J 36:3250-3267. https://doi.org/10.15252/embj.201796794.

19. Billker $O$, Lindo V, Panico M, Etienne AE, Paxton T, Dell A, Rogers $M$, Sinden RE, Morris HR. 1998. Identification of xanthurenic acid as the putative inducer of malaria development in the mosquito. Nature 392:289-292. https://doi .org/10.1038/32667.

20. Muhia DK, Swales CA, Deng W, Kelly JM, Baker DA. 2001. The gametocyteactivating factor xanthurenic acid stimulates an increase in membraneassociated guanylyl cyclase activity in the human malaria parasite Plasmodium falciparum. Mol Microbiol 42:553-560. https://doi.org/10.1046/j .1365-2958.2001.02665.x.

21. McRobert L, Taylor CJ, Deng W, Fivelman QL, Cummings RM, Polley SD Billker O, Baker DA. 2008. Gametogenesis in malaria parasites is mediated by the cGMP-dependent protein kinase. PLoS Biol 6:e139. https://doi.org/ 10.1371/journal.pbio.0060139.

22. Brochet M, Collins MO, Smith TK, Thompson E, Sebastian S, Volkmann K, Schwach F, Chappell L, Gomes AR, Berriman M, Rayner JC, Baker DA, Choudhary J, Billker O. 2014. Phosphoinositide metabolism links CGMPdependent protein kinase $\mathrm{G}$ to essential $\mathrm{Ca}^{2+}$ signals at key decision points in the life cycle of malaria parasites. PLoS Biol 12:e1001806. https://doi.org/10.1371/journal.pbio.1001806.

23. Taylor CJ, McRobert L, Baker DA. 2008. Disruption of a Plasmodium falciparum cyclic nucleotide phosphodiesterase gene causes aberrant gametogenesis. Mol Microbiol 69:110-118. https://doi.org/10.1111/j.1365-2958 2008.06267.x

24. Hirai M, Arai M, Kawai S, Matsuoka H. 2006. PbGC $\beta$ is essential for Plasmodium ookinete motility to invade midgut cell and for successful completion of parasite life cycle in mosquitoes. J Biochem 140:747-757. https:// doi.org/10.1093/jb/mvj205.

25. Moon RW, Taylor CJ, Bex C, Schepers R, Goulding D, Janse CJ, Waters AP, Baker DA, Billker O. 2009. A cyclic GMP signalling module that regulates gliding motility in a malaria parasite. PLoS Pathog 5:e1000599. https://doi .org/10.1371/journal.ppat.1000599.

26. Lakshmanan V, Fishbaugher ME, Morrison B, Baldwin M, Macarulay M, Vaughan AM, Mikolajczak SA, Kappe SHI. 2015. Cyclic GMP balance is critical for malaria parasite transmission from the mosquito to the mammalian host. mBio 6:e02330-14. https://doi.org/10.1128/mBio.02330-14.

27. Govindasamy K, Jebiwott $S$, Jaijyan DK, Davidow A, Ojo KK, Van Voorhis WC, Brochet M, Billker O, Bhanot P. 2016. Invasion of hepatocytes by Plasmodium sporozoites requires cGMP-dependent protein kinase and calcium-dependent protein kinase 4. Mol Microbiol 102:349-363. https://doi .org/10.1111/mmi.13466.

28. Falae A, Combe A, Amaladoss A, Carvalho T, Menard R, Bhanot P. 2010 Role of Plasmodium berghei cGMP-dependent protein kinase in late liver stage development. J Biol Chem 285:3282-3288. https://doi.org/10.1074/ jbc.M109.070367.

29. Kenthirapalan S, Waters AP, Matuschewski K, Kooij TWA. 2016. Functional profiles of orphan membrane transporters in the life cycle of the malaria parasite. Nat Commun 7:10519. https://doi.org/10.1038/ncomms10519.

30. Taylor HM, McRobert L, Grainger M, Sicard A, Dluzewski AR, Hopp CS Holder AA, Baker DA. 2010. The malaria parasite cyclic GMP-dependent protein kinase plays a central role in blood-stage schizogony. Eukaryot Cell 9:37-45. https://doi.org/10.1128/EC.00186-09.

31. Collins CR, Hackett F, Strath M, Penzo M, Withers-Martinez C, Baker DA, Blackman MJ. 2013. Malaria parasite cGMP-dependent protein kinase regulates blood-stage merozoite secretory organelle discharge and egress. PLoS Pathog 9:e1003344. https://doi.org/10.1371/journal.ppat.1003344.

32. Thomas JA, Tan MSY, Bisson C, Borg A, Umrekar TR, Hackett F, Hale VL, Vizcay-Barrena G, Fleck RA, Snijders AP, Saibil HR, Blackman MJ. 2018. A protease cascade regulates release of the human malaria parasite Plasmodium falciparum from host red blood cells. Nat Microbiol 3:447-455. https://doi.org/10.1038/s41564-018-0111-0.

33. Alam MM, Solyakov L, Bottrill AR, Flueck C, Siddiqui FA, Singh S, Mistry S, Viskaduraki M, Lee K, Hopp CS, Chitnis CE, Doerig C, Moon RW, Green JL,
Holder AA, Baker DA, Tobin AB. 2015. Phosphoproteomics reveals malaria parasite protein kinase $G$ as a signalling hub regulating egress and invasion. Nat Commun 6:7285. https://doi.org/10.1038/ncomms8285.

34. Koussis K, Withers-Martinez C, Baker DA, Blackman MJ. 2020. Simultaneous multiple allelic replacement in the malaria parasite enables dissection of PKG function. Life Sci Alliance 3:e201900626. https://doi.org/10.26508/ Isa.201900626.

35. Collins CR, Das S, Wong EH, Andenmatten N, Stallmach R, Hackett $F$ Herman J-P, Müller S, Meissner M, Blackman MJ. 2013. Robust inducible Cre recombinase activity in the human malaria parasite Plasmodium falciparum enables efficient gene deletion within a single asexual erythrocytic growth cycle. Mol Microbiol 88:687-701. https://doi.org/10.1111/ mmi.12206

36. Jones ML, Das S, Belda H, Collins CR, Blackman MJ, Treeck M. 2016. A versatile strategy for rapid conditional genome engineering using loxP sites in a small synthetic intron in Plasmodium falciparum. Sci Rep 6:21800. https://doi.org/10.1038/srep21800.

37. Byun JA, Van K, Huang J, Henning P, Franz E, Akimoto M, Herberg FW, Kim C. 2020. Mechanism of allosteric inhibition in the Plasmodium falciparum cGMP-dependent protein kinase. J Biol Chem 295:8480-8491. https://doi .org/10.1074/jbc.RA120.013070.

38. Salowe SP, Wiltsie J, Liberator PA, Donald RGK. 2002. The role of a parasite-specific allosteric site in the distinctive activation behavior of Eimeria tenella CGMP-dependent protein kinase. Biochemistry 41:4385-4391. https://doi.org/10.1021/bi0156658.

39. Lutsenko S, Kaplan JH. 1995. Organization of P-type ATPases: significance of structural diversity. Biochemistry 34:15607-15613. https://doi.org/10 .1021/bi00048a001.

40. Kuhlbrandt W. 2004. Biology, structure, and mechanism of P-type ATPases. Nat Rev Mol Cell Biol 5:282-295. https://doi.org/10.1038/nrm1354.

41. Montigny C, Lyons J, Champeil P, Nissen P, Lenoir G. 2016. On the molecular mechanism of flippase- and scramblase-mediated phospholipid transport. Biochim Biophys Acta 1861:767-783. https://doi.org/10.1016/ j.bbalip.2015.12.020.

42. Chen CY, Ingram MF, Rosal PH, Graham TR. 1999. Role for Drs2p, a P-type ATPase and potential aminophospholipid translocase, in yeast late Golgi function. J Cell Biol 147:1223-1236. https://doi.org/10.1083/jcb.147.6 .1223.

43. Hopp CS, Flueck C, Solyakov L, Tobin A, Baker DA. 2012. Spatiotempora and functional characterization of the Plasmodium falciparum cGMP-dependent protein kinase. PLoS One 7:e48206. https://doi.org/10.1371/ journal.pone.0048206.

44. Jiang Y, Wei J, Cui H, Liu C, Zhi Y, Jiang Z, Li Z, Li S, Yang Z, Wang X, Qian P, Zhang C, Zhong C, Su X-Z, Yuan J. 2020. An intracellular membrane protein GEP1 regulates xanthurenic acid induced gametogenesis of malaria parasites. Nat Commun 11:1764. https://doi.org/10.1038/s41467-020 -15479-3.

45. Zhang M, Wang C, Otto TD, Oberstaller J, Liao X, Adapa SR, Udenze K, Bronner IF, Casandra D, Mayho M, Brown J, Li S, Swanson J, Rayner JC, Jiang RHY, Adams JH. 2018. Uncovering the essential genes of the human malaria parasite Plasmodium falciparum by saturation mutagenesis. Science 360:eaap7847. https://doi.org/10.1126/science.aap7847.

46. Bushell E, Gomes AR, Sanderson T, Anar B, Girling G, Herd C, Metcalf T, Modrzynska K, Schwach F, Martin RE, Mather MW, McFadden Gl, Parts L, Rutledge GG, Vaidya AB, Wengelnik K, Rayner JC, Billker O. 2017. Functional profiling of a Plasmodium genome reveals an abundance of essential genes. Cell 170:260-272 e8. https://doi.org/10.1016/j.cell.2017.06.030.

47. Moudy R, Manning TJ, Beckers CJ. 2001. The loss of cytoplasmic potassium upon host cell breakdown triggers egress of Toxoplasma gondii. J Biol Chem 276:41492-41501. https://doi.org/10.1074/jbc.M106154200.

48. Roiko MS, Svezhova N, Carruthers VB. 2014. Acidification activates Toxoplasma gondii motility and egress by enhancing protein secretion and cytolytic activity. PLoS Pathog 10:e1004488. https://doi.org/10.1371/journal .ppat.1004488.

49. Patel A, Perrin AJ, Flynn HR, Bisson C, Withers-Martinez C, Treeck M, Flueck C, Nicastro G, Martin SR, Ramos A, Gilberger TW, Snijders AP, Blackman MJ, Baker DA. 2019. Cyclic AMP signalling controls key components of malaria parasite host cell invasion machinery. PLoS Biol 17:e3000264. https://doi .org/10.1371/journal.pbio.3000264.

50. Zhou Z, Sayed N, Pyriochou A, Roussos C, Fulton D, Beuve A, Papapetropoulos A. 2008. Protein kinase $G$ phosphorylates soluble guanylyl cyclase on serine 64 and inhibits its activity. Arterioscler Thromb Vasc Biol 28:1803-1810. https:// doi.org/10.1161/ATVBAHA.108.165043.

51. Roland BP, Graham TR. 2016. Decoding P4-ATPase substrate interactions. 
Crit Rev Biochem Mol Biol 51:513-527. https://doi.org/10.1080/10409238 .2016.1237934.

52. Paul AS, Miliu A, Paulo JA, Goldberg JM, Bonilla AM, Berry L, Seveno M, Braun-Breton C, Kosber AL, Elsworth B, Arriola JSN, Lebrun M, Gygi SP, Lamarque MH, Duraisingh MT. 2020. Co-option of Plasmodium falciparum PP1 for egress from host erythrocytes. Nat Commun 11:3532. https://doi .org/10.1038/s41467-020-17306-1.

53. Woehlbier U, Epp C, Kauth CW, Lutz R, Long CA, Coulibaly B, Kouyaté B, Arevalo-Herrera M, Herrera S, Bujard H. 2006. Analysis of antibodies directed against merozoite surface protein 1 of the human malaria parasite Plasmodium falciparum. Infect Immun 74:1313-1322. https://doi.org/ 10.1128/IAI.74.2.1313-1322.2006.

54. Collins CR, Withers-Martinez C, Hackett F, Blackman MJ. 2009. An inhibitory antibody blocks interactions between components of the malarial invasion machinery. PLoS Pathog 5:e1000273. https://doi.org/10.1371/ journal.ppat.1000273.

55. Stallmach R, Kavishwar M, Withers-Martinez C, Hackett $F$, Collins $C R$, Howell SA, Yeoh S, Knuepfer E, Atid AJ, Holder AA, Blackman MJ. 2015. Plasmodium falciparum SERA5 plays a non-enzymatic role in the malarial asexual blood-stage lifecycle. Mol Microbiol 96:368-387. https://doi.org/ $10.1111 / \mathrm{mmi} .12941$

56. Trager W, Jensen JB. 1976. Human malaria parasites in continuous culture. Science 193:673-675. https://doi.org/10.1126/science.781840.
57. Lambros C, Vanderberg JP. 1979. Synchronization of Plasmodium falciparum erythrocytic stages in culture. J Parasitol 65:418-420. https://doi.org/ $10.2307 / 3280287$.

58. Thomas JA, Collins CR, Das S, Hackett F, Graindorge A, Bell D, Deu E, Blackman MJ. 2016. Development and application of a simple plaque assay for the human malaria parasite Plasmodium falciparum. PLoS One 11:e0157873. https://doi.org/10.1371/journal.pone.0157873.

59. Smilkstein M, Sriwilaijaroen N, Kelly JX, Wilairat P, Riscoe M. 2004. Simple and inexpensive fluorescence-based technique for high-throughput antimalarial drug screening. Antimicrob Agents Chemother 48:1803-1806. https://doi.org/10.1128/aac.48.5.1803-1806.2004.

60. Das S, Hertrich N, Perrin AJ, Withers-Martinez C, Collins CR, Jones ML, Watermeyer JM, Fobes ET, Martin SR, Saibil HR, Wright GJ, Treeck M, Epp C, Blackman MJ. 2015. Processing of Plasmodium falciparum merozoite surface protein MSP1 activates a spectrin-binding function enabling parasite egress from RBCs. Cell Host Microbe 18:433-444. https://doi.org/10 .1016/j.chom.2015.09.007.

61. Kawamoto F, Alejo-Blanco R, Fleck SL, Kawamoto Y, Sinden RE. 1990. Possible roles of $\mathrm{Ca} 2+$ and CGMP as mediators of the exflagellation of Plasmodium berghei and Plasmodium falciparum. Mol Biochem Parasitol 42:101-108. https://doi.org/10.1016/0166-6851(90)90117-5. 